\title{
Study of synaptic vesicle cycling during exo- and endocytosis using optical approaches
}

\author{
Dissertation \\ for the award of the degree \\ "Doctor rerum naturalium" \\ of the Georg-August-Universität Göttingen
}

submitted by

Yunfeng Hua

from Shanghai, PR China

Göttingen 2012 
Prof. Dr. Erwin Neher

Dept. Membrane Biophysics, Max-Planck-Institute of Biophysical Chemistry

Prof. Dr. Jürgen Klingauf

Dept. Cellular Biophysics, Institute of Medical Physics and Biophysics, University Münster

Dr. Alexander Egner

Laser-Laboratorium Göttingen e.V. 


\section{Declaration}

I hereby declare that my doctoral thesis 'study of synaptic vesicle cycling during exo- and endocytosis using optical approaches' has been written independently with no other aids or sources than quoted.

Yunfeng Hua 


\section{Contents}

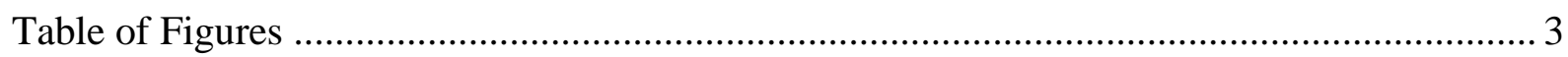

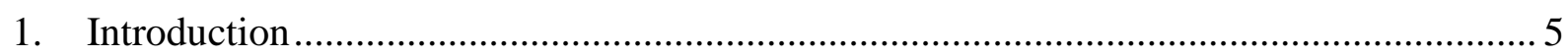

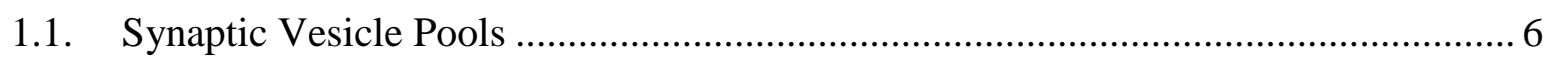

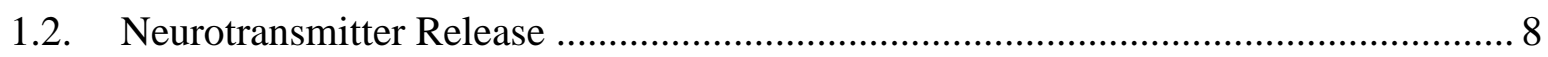

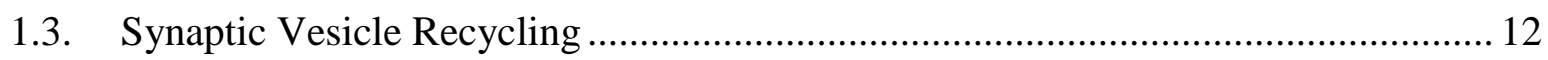

1.4. Readily Retrievable Surface Pool........................................................................ 16

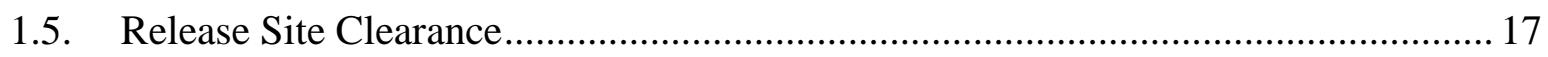

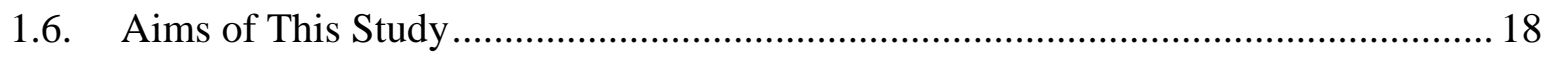

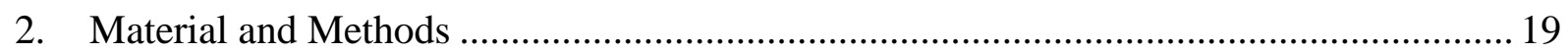

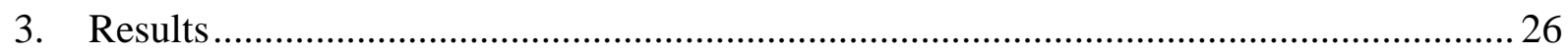

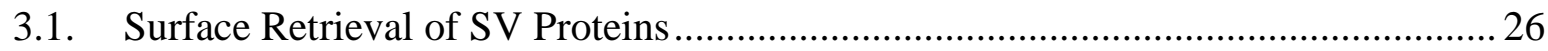

3.2. Exo-endocytosis Cycling of Endogenous SV Proteins............................................. 29

3.3. Spatial Organization of the Readily Retrievable Pool ............................................... 31

3.4. Quantitative Measurement of Cumulative Release ................................................... 34

3.5. Limited Vesicle Reuse during Mild Stimulation ....................................................... 39

3.6. Blocking Endocytosis Causes Rapid Release Depression.......................................... 41

3.7. Vesicle Recruitment during Multiple Spaced Stimuli ............................................... 44

3.8. Surface Accumulation of SV Components Caused by Dynasore............................... 46

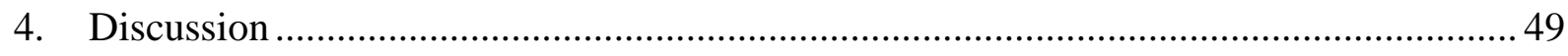

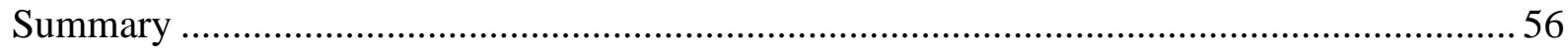




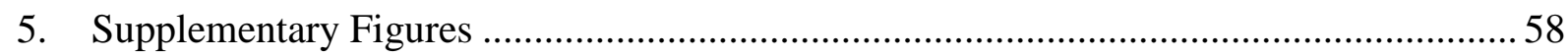

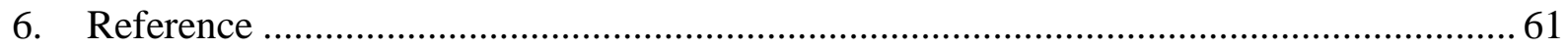

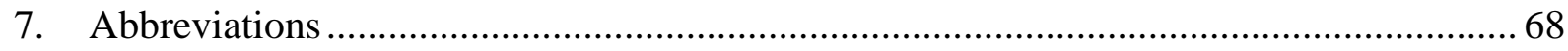

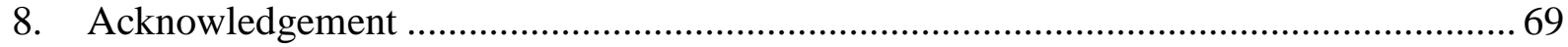

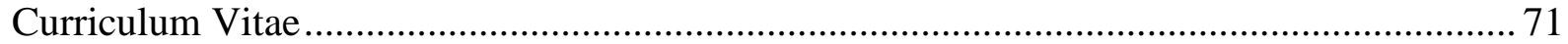

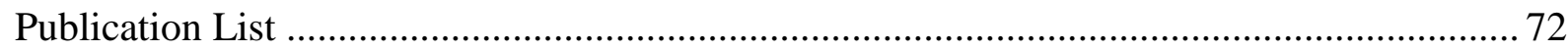




\section{Table of Figures}

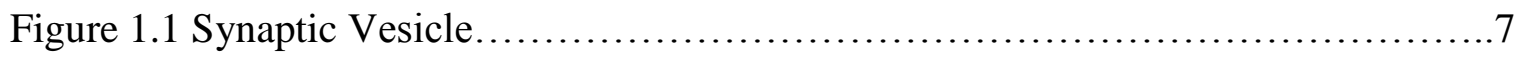

Figure 1.2 SNARE Complexes and Neuroexocytosis..............................9

Figure 1.3 Imaging SV Recycling with pHluorin-based Probes.........................11

Figure 1.4 Different Modes of SV Recycling...................................13

Figure 2.1 Photobleaching Correction for cypHer Signals..........................24

Figure 3.1 cypHer-coupled Antibodies Are a Reliable Tool to Measure Exo-endocytic

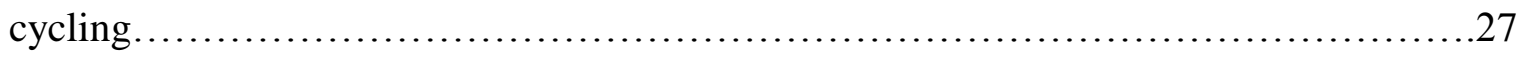

Figure 3.2 Readily Retrievable Surface Pool of SV Components.....................28

Figure 3.3 Comparison of Vesicle Recycling Kinetics Probed with spH and cypHercoupled Antibodies....................................................... 30

Figure 3.4 Spatial Organization of the RRetP.................................. 33

Figure 3.5 Normalization of spH Response Using a Paired-stimulation Protocol...........36

Figure 3.6 Color-coded Endocytic Time Constants of Synapses Challenged with Different Stimulation Protocols.......................................................... 37

Figure 3.7 Quantitative Measurement of Cumulative Release...........................38

Figure 3.8 Same Amount of Vesicles Participating in Exocytosis during Mild Stimulation

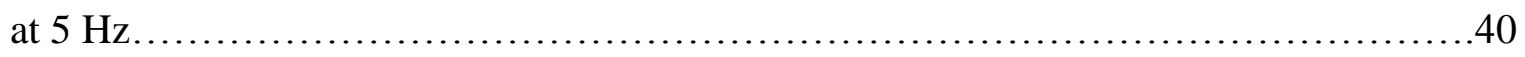

Figure 3.9 Frequency-dependent Release Depression in the Presence of Dynasore.......42

Figure 3.10 Reacidification has no Effect on Dynasore-enhanced Release Depression...43

Figure 3.11 Recruitment of Reserve Vesicles during Multiple Spaced Stimuli...........45

Figure 3.12 Acuumulation of Vesicle Components in the Presence of Dynasore..........48 
Suppl. 1 Intracellular Calcium Level Regulates the Recycling Pool Size................58

Suppl. 2 Dynasore-enhanced Release Depression Probed by cypHer..................59

Suppl. 3 Live Staining of Newly Exocytosed SV Proteins..........................60 


\section{Introduction}

In the central nervous system, communication between nerve cells mainly occurs at specialized junctions called synapses. At the chemical synapse, electrical signals trigger controlled secretion of neurotransmitter via exocytosis of synaptic vesicles (SV) at the presynaptic site. The fusion of SVs releases neurotransmitters that diffuse across the synaptic cleft and activate postsynaptic receptor channels. That generates a new wave of

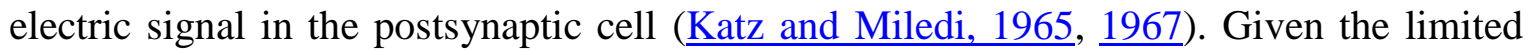
number of SVs in a presynaptic terminal, complete fusion of many SVs with the plasma membrane would result in fast depletion of fusion-competent SVs and thereby the loss of the ability to transmit signals across synapses. Therefore, to support rapid and repeated rounds of release, a fast trafficking cycle of SVs is required to maintain synaptic function during sustained synaptic activities (Harata et al., 2001; Schweizer and Ryan, 2006; Sudhof, 2004). Although intense research over three decades has led to a deep understanding of the molecular mechanisms behind exocytic membrane fusion (Jahn and $\underline{\text { Scheller, 2006; Martens and McMahon, 2008) and endocytic retrieval of SV (Dittman and }}$ Ryan, 2009; Doherty and McMahon, 2009), comparatively little is known about the coupling between exo- and endocytosis. Hence, understanding the underlying mechanisms of the interplay between exo- and endocytosis still remains one of the central challenges of presynaptic physiology. 


\subsection{Synaptic Vesicle Pools}

SVs are unique secretory organelles with an average diameter of $42 \mathrm{~nm}$. Each SV contains about 1500-2000 neurotransmitter molecules. Through proteomic and lipidomic analysis approximately 40 integral membrane proteins have been identified to be vesicle residents (Figure 1.1), some of which are present in surprisingly low copy numbers per vesicle (Takamori et al., 2006). At each small synaptic bouton $(\sim 1 \mu \mathrm{m}$ in diameter) of hippocampal primary neurons, the total vesicle population comprises about 200 vesicles (Figure 1.1). These vesicles consist of almost the same components and are clustered in a phosphorylation-dependent manner at the site of neurotransmitter release (Greengard et al., 1993; Schikorski and Stevens, 1997).

According to their release kinetics, SVs can be assigned to three different subgroups (Harata et al., 2001; Li et al., 2005; Rizzoli and Betz, 2005; Schikorski and Stevens, 2001). Vesicles of the readily releasable pool (RRP, about $5 \%$ of total population) are those spatially docked to the site of release and fuse immediately upon stimulation. Those vesicles recruited to the release site upon moderate physiological stimulation comprise the recycling pool (about $45 \%$ of total population), which is refilled by newly endocytosed vesicles after stimulation. The rest of vesicles comprise the resting pool, as those vesicles do not seem to cycle even upon exhaustive stimulation. Potential physiological functions of this pool are proposed to control presynaptic homeostasis during neuronal development (Andreae et al., 2012) and maintain high concentration of soluble proteins in the nerve terminals (Denker et al., 2011). These three vesicle pools are not anatomically segregated and mobilization of vesicles between different pools is possible (Rizzoli and Betz, 2005). Moreover, a recent study suggested that the size of the recycling and resting vesicle pools might be dynamically regulated by cyclin-dependent kinase 5 (CDK5) and calcineurin 
(CN) activity, providing a potent control system for tuning synaptic performance (Kim and Ryan, 2010).
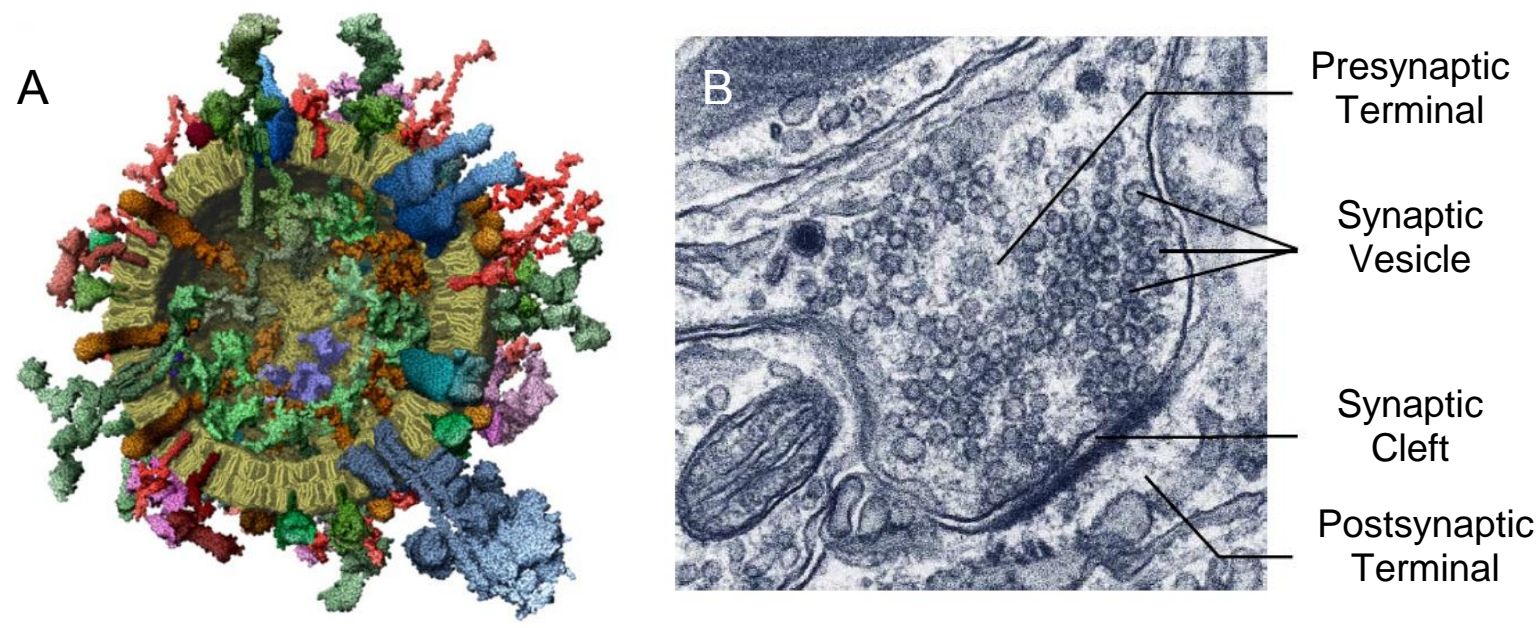

Figure 1.1 Synaptic Vesicle

(A) Molecular model of an average SV. Taken from (Takamori et al., 2006)

(B) Electron microscopic image of a chemical synapse on rat hippocampal neurons. 


\subsection{Neurotransmitter Release}

SNARE proteins and neuroexocytosis. Upon arrival of an electrical impulse (action potential, AP) at the presynapse, membrane depolarization induces the opening of voltagegated calcium channels (VGCC) that are clustered at specialized regions, called active zones (AZs). In the vicinity of these open channels localized high calcium concentrations (so-called $\mathrm{Ca}^{2+}$ nanodomains) are created and trigger the fusion of docked and primed SVs with plasma membrane, releasing their chemical content into the synaptic cleft (Rizo and Rosenmund, 2008).

SV fusion during neuroexocytosis is the fastest form of intracellular membrane fusion. A key element of this process is the formation of a complex between components in the vesicular and target plasma membranes, the so-called SNARE (soluble NSF (Nethylmaleimide-sensitive factor) attachment protein receptor) proteins (Figure 1.2). Once the complex is formed, its conformational change provides the driving force required for the fusion of the two membranes (Sudhof and Rothman, 2009). As suggested in several recent studies only few SNARE complexes are sufficient to accomplish vesicle fusion (Mohrmann et al., 2010; Sinha et al., 2011; van den Bogaart et al., 2010). However, to release neurotransmitter in a spatial and temporally defined manner, fusion of vesicles needs to be tightly controlled by the development of $\mathrm{Ca}^{2+}$ nanodomains that are sensed by the vesicle associated protein synaptotagmin 1 (Syt1) through its $\mathrm{Ca}^{2+}$-binding $\mathrm{C} 2$ domains. Indeed, it was found that neurons lacking Syt1 display complete absence of fast synchronized release (Geppert et al., 1994). Despite its important role in $\mathrm{Ca}^{2+}$-triggered exocytosis, it still remains controversial whether Syt1 acts on SNARE complexes physically (Dai et al., 2007; Lai et al., 2011) or just serves as a distance regulator of SNARE complex formation (van den Bogaart et al., 2011). 


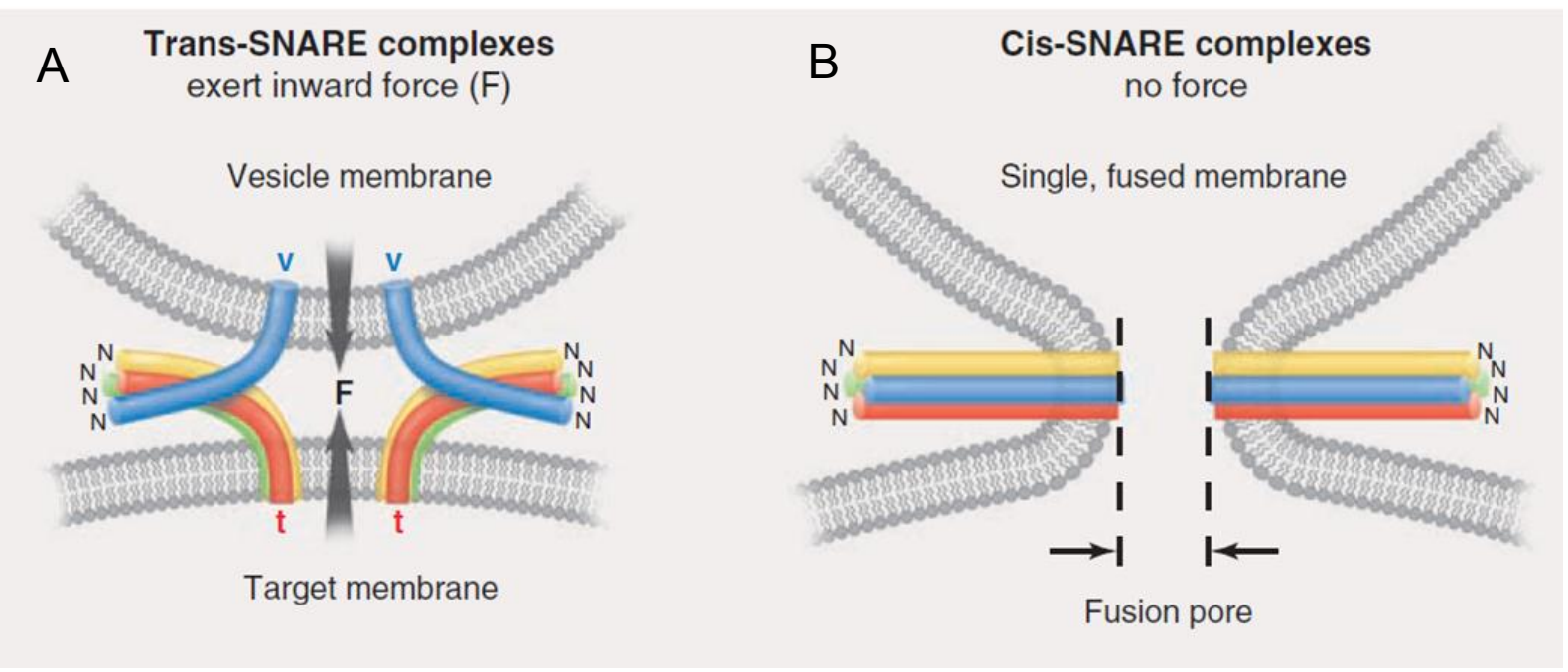

Figure 1.2 SNARE Complexes and Neuroexocytosis

(A) The zippering model for SNARE-catalyzed membrane fusion. Assembly proceeds progressively from the membrane-distal $\mathrm{N}$ termini toward the membrane-proximal $\mathrm{C}$ terminal of the SNAREs. This generates an inward force that pulls the bilayers together, forcing them to fuse.

(B) When fusion has occurred, complete zippering turns the SNAREs in the low-energy cis-conformation. Taken from (Sudhof and Rothman, 2009).

Multiple roles of calcium ions in the regulation of neurotransmitter release. The intracellular $\mathrm{Ca}^{2+}$ concentration not only has an important role in the triggering of neurotransmitter release but also in the regulation of short-term synaptic plasticity (STP) (Neher and Sakaba, 2008). A short burst of neurotransmitter release is initiated by high $\mathrm{Ca}^{2+}$ concentration within nanodomains near VGCCs and can be electrophysiologically registered as a single peak during recording of excitatory postsynaptic current (EPSC). During repetitive presynaptic activities, however, the measured EPSC amplitudes drop progressively and reach a steady-state level within 10-20 stimuli, indicating a reduced rate 
of neurotransmitter release. This phenomenon is known as short-term synaptic depression (STD) and is expected to modulate information processing in neuronal networks of the central nervous system by influencing the synaptic output during repetitive activity. A candidate mechanism for the induction of STD is depletion of vesicles in the RRP and the following steady-state is characterized by a balance of SV consumption and repopulation in this pool from a more distant reserve pool. During sustained synaptic activity, however, the recruitment of release-ready vesicles can be accelerated with a global rise in calcium concentration (Wang and Kaczmarek, 1998) and controls the level of STD as well as the recovery of the vesicle pool in periods of rest in an activity-dependent manner (Neher and

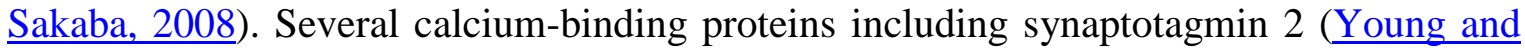

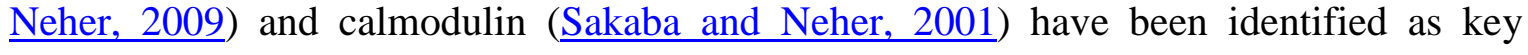
players in calcium-dependent vesicle replenishment by affecting important steps like vesicle docking and priming near VGCCs. In addition, it was reported recently that the intracellular $\mathrm{Ca}^{2+}$ level during stimulation also regulates the recycling pool size in the presynaptic bouton of rat hippocampal neuron ( $\mathrm{Li}$ et al., 2011), suggesting an important role of calcium ions in dynamic regulation of synaptic strength.

Visualization of vesicle fusion using a pH-reporter. Recently, $\mathrm{pH}$-sensitive GFP (pHluorin) has been used to visualize secretion at presynaptic boutons (Miesenbock et al., 1998). When tagged to the luminal domain of SV associated proteins like SV SNARE synaptobrevin 2 (Syb 2) or vesicular glutamate transporter (vGlut), pHluorin can be successfully introduced to the SV lumen upon overexpression in neurons. Inside the SV lumen, with a typical $\mathrm{pH}$ of 5.6, the fluorescence of $\mathrm{spH}$ molecules is almost completely quenched. Upon exposure to the extracellular environment and a neutral $\mathrm{pH}$ of 7.4 during exocytosis, each $\mathrm{spH}$ molecule gains a $\sim 20$-fold increase in fluorescence (Figure 1.3). 
Using these $\mathrm{pH}$-dependent changes in fluorescence intensities, it is possible to measure stimulation-dependent exo- and endocytosis in real time in presynaptic boutons of neurons (Sankaranarayanan et al., 2000; Sankaranarayanan and Ryan, 2000). In the past ten years, these genetically encoded reporters have been extensively used to study kinetics of vesicle recycling (Balaji and Ryan, 2007; Royle et al., 2008).

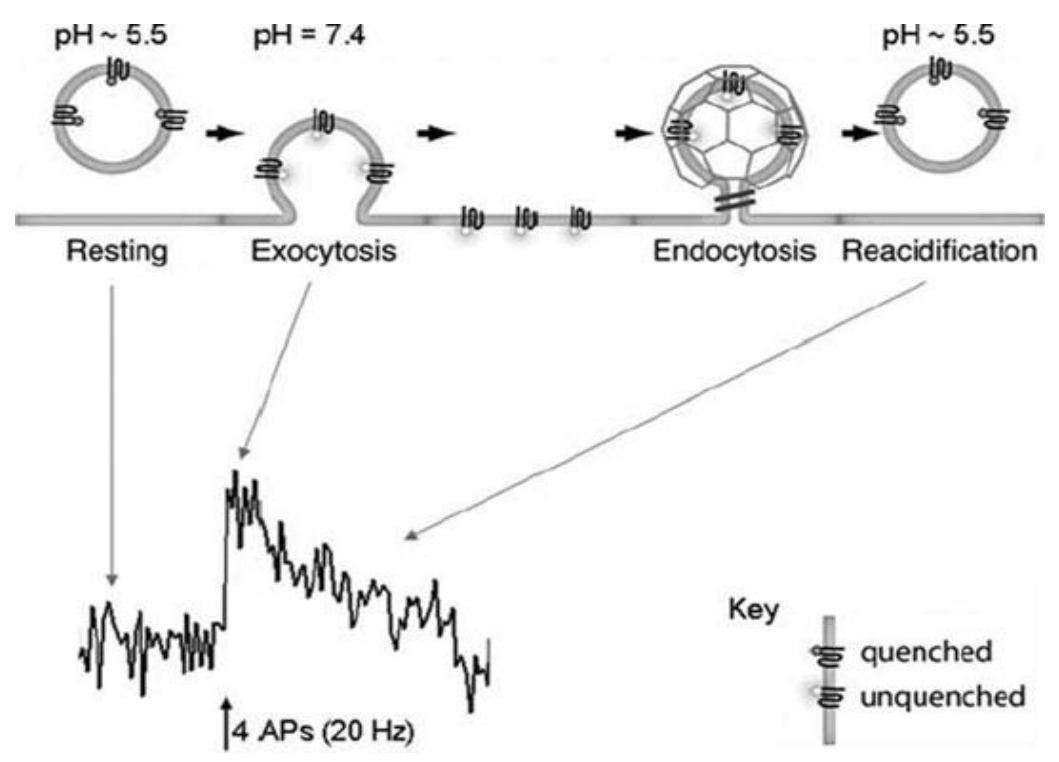

Figure 1.3 Imaging SV Recycling with pHluorin-based Probes

The pHluorin is quenched by the low $\mathrm{pH}$ inside the vesicle lumen. Upon stimulation (black arrow), vesicle fuses with the membrane and deprotonation switches the pHluorin in to its fluorescent form (upward deflection, in the trace, black). The fluorescence drops to the base line following endocytosis and vesicle reacidification. Taken from (Royle et al., 2008). 


\subsection{Synaptic Vesicle Recycling}

Mulitple vesicle retrieval pathways. After membrane fusion, compensatory endocytosis is required for cell to maintain constant surface area by specifically retrieving membrane and proteins inserted by calcium-triggered exocytosis (Dittman and Ryan, 2009; Murthy and De Camilli, 2003). The mechanism of the retrieval is comparatively more controversial than that of the fusion, although it occurs at longer time scales and can be more easily studied. Evidence from electron micrographs reveal that newly formed vesicles are mainly found at specialized region called endocytic site, which surrounds the presynaptic AZ (Heuser and Reese, 1973; Miller and Heuser, 1984). After having been refilled with neurotransmitter, those vesicles are ready for a next round of exocytosis. Multiple vesicle retrieval pathways might coexist with different characteristic time constants (Gandhi and

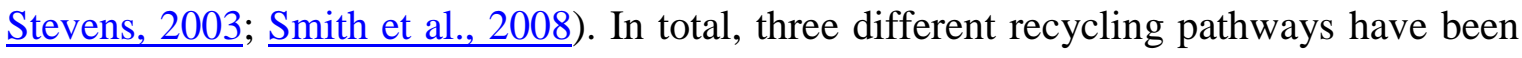
proposed (Figure 1.4). A fast mode called 'kiss and run' describes neurotransmitter release via a narrow fusion pore during a brief connection between the SV and the plasma membrane. Rapid closure of the pore enables the vesicle being retrieved intact within one second (Aravanis et al., 2003; Klingauf et al., 1998; Pyle and Pelletier, 2003; Zhang et al., 2009). The more classical model proposed by Heuser and Reese involves the full fusion and collapse of the SV followed by clathrin-mediated endocytosis (CME) and is thought to be the major pathway for SV recycling. These coated vesicles are derived from invaginations of the plasma membrane which later bud off, forming a nascent SV for successive rounds of exocytosis (Granseth et al., 2006; Heuser and Reese, 1973). According to this mechanism, SV collapse and retrieval occur with a time constant of about tens of seconds. A third pathway, called bulk endocytosis, is observed during heavy stimulation when large membrane infoldings appear which bud off vesicles via CME (Koenig and Ikeda, 1996; Takei et al., 1996). Although having been intensively studied 
for many years, some aspects of this topic still remain unclear, including whether different pathways produce functionally different pools of vesicles and what role the different modes may have on neurotransmission (Kavalali, 2007; Voglmaier and Edwards, 2007). Furthermore, a recent study in the mouse motor nerve terminals suggests a change in the location of preferred endocytic sites upon different stimulation strengths, suggesting a possible stimulus-dependent modulation of retrieval pathways (Gaffield et al., 2009).

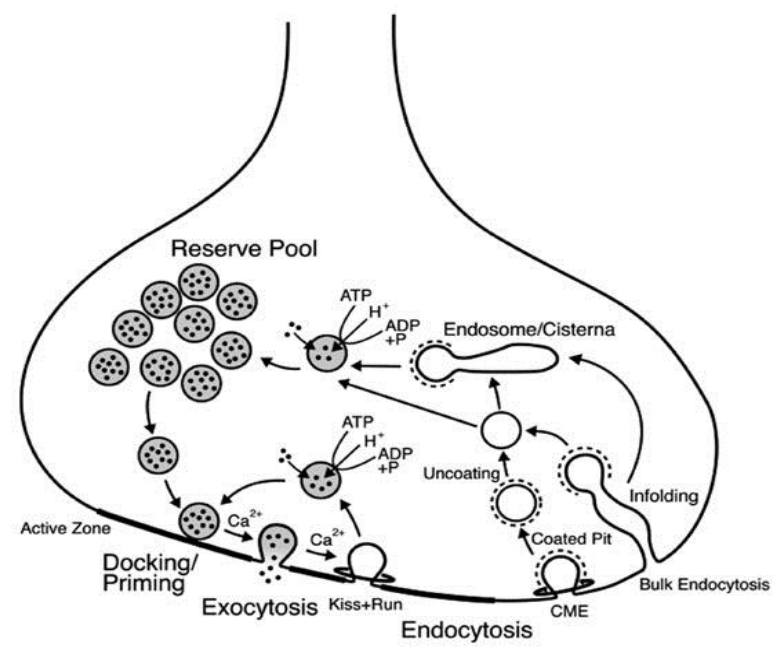

Figure 1.4 Different Modes of SV Recycling

Schematic illustration of different modes of SV recycling. A fast 'kiss-and-run' mechanism, where the vesicle connects only briefly to the plasma membrane without full collapse ("kiss \& run"), a slow pathway via large infoldings and endosomes ("bulk endocytosis"), and recovery of vesicle membrane by clathrin-coated pits (clathrinmediated endocytosis, "CME"). Taken from (Haucke and Klingauf, 2006). 
Modulation of endocytosis: calcium and exocytic load. Quantitative estimates of membrane retrieval after exocytosis provide information about roles of modulated endocytic pathways. Two different techniques are widely used. Whole-cell capacitance measurements, in which real-time changes of cell surface area are monitored by measuring the membrane capacitance with great temporal resolution through patch-clamp pipettes, are well established in studies of large nerve terminals like Calyx of Held (Augustine and Neher, 1992; Sun and Wu, 2001; von Gersdorff and Matthews, 1994). The second technique involves fluorescent optical tracers at small synapses and enables us mapping in a large scale the key feature of membrane or vesicle protein uptake ( al., 1993; Sankaranarayanan and Ryan, 2000). The appearance of distinct kinetic components observed from both techniques has been interpreted as participation of different retrieval modes. Interestingly, aside from the importance in signaling, the cytosolic calcium level at nerve terminal seems to have a role in regulation of endocytosis as well. In most types of synapses in the central nervous system, intracellular calcium elevation leads to accelerated retrieval of vesicular proteins (Sankaranarayanan and Ryan, 2001; Wu et al., 2005). However, an inhibitory effect of calcium on endocytosis has also been uncovered in the retinal bipolar cell terminal (von Gersdorff and Matthews, 1994). However the functional role of such inhibition under physiological conditions is still unclear. In addition, the progressive decline of the rate of endocytosis has been consistently observed after prolonged electrical stimulation in hippocampal synaptic boutons, despite the fact that endocytosis was initially accelerated under conditions that led to elevated intracellular calcium concentration. Thus, it has been suggested that this slowing of endocytosis with the accumulation of vesicle components may result from saturation of the total endocytic capacity of the synapse (Balaji et al., 2008; Sankaranarayanan and Ryan, 2001). 
Coupling of exo- and endocytosis. The spatial and temporal coupling of exocytic membrane fusion and compensatory retrieval is a crucial and central element in the maintenance of synaptic transmission in the central nervous system during sustained synaptic activity. Without compensatory retrieval, continuous fusion of SVs with the plasma membrane would lead to a fast depletion of the SV pool and expansion of the presynaptic membrane. Evidence for such functional coupling includes the dual function of many SV proteins (e.g. Syb 2, Syt 1 or synaptophysin) that were originally considered to play a role exclusively in exocytosis, but have been found to have an additional role in compensatory endocytosis (Daly et al., 2000; Deak et al., 2004; Kwon and Chapman, 2011; Nicholson-Tomishima and Ryan, 2004; Poskanzer et al., 2003). Additionally, interfering with the function of endocytic proteins (e.g. dynamin) leads to a strong depression of exocytosis (Hosoi et al., 2009; Kawasaki et al., 2000). The local intracellular calcium concentration, which rises and falls rapidly during an AP, seems to serve as a central regulatory factor in these processes. During stimulation, high concentrations of calcium not only trigger exocytosis, but also facilitate endocytosis (Sankaranarayanan and Ryan, 2001). Recently, the SV associated transmembrane protein known as 'flower' (a proposed calcium channel) was shown to regulate SV endocytosis, and thus, potentially controls exo-endocytic coupling (Yao et al., 2009). These data imply a crucial role of vesicular proteins in regulating synaptic plasticity by shifting the balance between exo- and endocytosis during sustained synaptic activities. However, the underlying mechanisms remain controversial, probably due to the complexity emerging from the involvement of the multiple recycling pathways discussed above. 


\subsection{Readily Retrievable Surface Pool}

An unexpected finding from studies using the $\mathrm{spH}$ assay (see above) was that up to $30 \%$ of overexpressed probes are localized on the plasma membrane even under resting conditions (Fernandez-Alfonso et al., 2006; Wienisch and Klingauf, 2006). A minor surface expression of synaptophysin-pHluorin ( $8 \%)$ (Granseth et al., 2006) and vGlut1pHluorin $(\sim 2 \%$ ) (Balaji and Ryan, 2007) was also reported recently. This implies the existence of a pool of vesicular proteins stranded on the membrane surface and raises questions about the reason why these vesicular proteins are excluded during compensatory endocytosis. To explore the function of these surface-stranded SV proteins, their pHluorin-tags were selectively silenced through either proteolysis or photo-bleaching, while those in the vesicle lumens remained intact. Both manipulations resulted in a normal exocytosis-driven fluorescence increase followed by an impaired fluorescence decay, suggesting that newly exocytosed vesicular proteins exchange with surface-stranded ones during brief stimulation (Wienisch and Klingauf, 2006). Based on these observations, it was speculated that these membrane-stranded vesicular proteins may support the first wave of compensatory endocytosis and thereby participate actively in exo-endocytic cycling. Further evidence for a functional pool of preassembled SV components at the presynaptic membrane came from experiments using botulinum toxin (BoNT/A), which completely abolishes exocytosis by cleaving the membrane SNARE SNAP-25, but did not block endocytosis completely (Neale et al., 1999). This clearly suggests an exocytosisindependent retrieval of SV components from a pre-existing pool on the presynaptic membrane. If such a 'readily retrieval pool' (RRetP) of vesicle components exists, it may serve as a coupling factor between exo- and endocytosis through dynamic control of the pool size. Thus, it is crucial to optically resolve this functional pool and understand how it may couple exo- and endocytosis in a temporal and spatial defined manner. 


\subsection{Release Site Clearance}

It is well established that in many types of synapses the level of neurotransmitter release during ongoing activity is mainly limited by the availability of release-ready vesicles and, therefore, depends on the speed of vesicle recruitment. Surprisingly, many synapses in the central nervous system can sustain synaptic activity even upon high frequency stimulation (Kopp-Scheinpflug et al., 2008; Lorteije et al., 2009; Rancz et al., 2007). It is evident that vesicles are required to undergo fast recycling to prevent depletion of the vesicle pool (Fernandez-Alfonso and Ryan, 2004; Sudhof, 2004). It has been reported in several recent studies that interfering with the function of endocytic proteins causes a fast, stimulusfrequency-dependent depression of vesicle exocytosis (Hosoi et al., 2009; $\underline{\text { Kawasaki et al., }}$ 2000). Some of these inhibitory effects developed so rapidly that they could not simply be explained by depletion of recycled vesicles. Therefore, it has been postulated that the respective manipulations affect a step in between exo- and endocytosis (Haucke et al., 2011; Neher, 2010). Translocation of SV components from sites of release to the endocytic sites may be a most likely candidate for this step. Acute accumulation of newly exocytosed vesicular proteins may cause a functional block of the previously used release site by interfering with the docking of subsequent vesicles. Knowing how these newly exocytosed vesicular proteins are removed from the release site may allow us to better understand the interplay between exo- and endocytosis and to gain a more accurate picture of neurotransmitter release. 


\subsection{Aims of This Study}

In this study I explored the usability of the genetically encoded $\mathrm{pH}$-sensitive fluorescence probe $\mathrm{spH}$ (Miesenbock et al., 1998; Sankaranarayanan et al., 2000) as well as a newly developed exogenous pH-indicator (Hua et al., 2010; Hua et al., 2011) to visualize exoand endocytosis in cultured rat hippocampal neurons. Using these probes, I tried to address several unresolved questions concerning SV recycling. First: Is there a preexisting pool (so-called RRetP) of endogenous vesicle components on the membrane surface and what is its location relative to the presynaptic AZ? Second: Are endogenous vesicular proteins also preferentially retrieved from this surface pool during compensatory endocytosis as implied by studies using overexpressed probes and is the retrieval from such putative RRetP kinetically faster? If the RRetP has only a limited capacity for fast endocytosis, exhaustion of this pool will lead to a reduction of the endocytic rate and thereby should cause massive protein accumulation on the membrane surface. Will this eventually result in functional block of the previously used release site and become the rate-limiting step during sustained synaptic activities? 


\section{Material and Methods}

Cell Culture. Primary neuronal cultures ( $5000-7500$ cells per cover slip) were prepared according to the revised protocol established by C. Rosenmund with minor modifications (Rosenmund and Stevens, 1996). Hippocampal CA1-CA3 regions were dissected from one-day-old Wistar rats under the microscope and collected in a tissue dish filled with Hank's Balanced Salt Solution (HBSS, Invitrogen) on ice. This tissue was then transferred into a falcon tube and the following procedures in preparation were performed under sterile conditions. After being bubbled with carbogen gas for 10-30 minutes, $1 \mathrm{ml}$ digestion solution containing 20-25 units Papain was added to the tissue culture. After 1hour incubation at $37{ }^{\circ} \mathrm{C}$, the tissue was then transferred to inhibition solution and incubated for 5 minutes. The dispersion of the tissue into individual neurons was completed in required amount of serum-free Neurobasal A Medium (NBA, Invitrogen) by triturating with Pasterur pipette. The suspension was diluted with primary medium of required volume. Finally the neurons were plated onto $18 \mathrm{~mm}$ cover slips (Assistant, REF 1001/18; 0.13-0.16 mm) coated with Matrigel (Beckton-Dickinson, Heidelberg, Germany) and placed in a 12 well plate (BD 353043 ). Neuronal cultures were kept in an incubator (Heraeus BBD 6620) at $37^{\circ} \mathrm{C}$ and $5 \% \mathrm{CO}_{2}$ before use (DIV 14-28).

Transfection of $\mathbf{s p H}$. Cultured neurons were transfected at 3 DIV by a modified calcium phosphate transfection procedure (Wienisch and Klingauf, 2006). First NBA solution was prewarmed 30-minute prior to the transfection. Calcium phosphate/DNA precipitates were then formed by mixing 5-30 $\mu \mathrm{g} / \mathrm{ml}$ plasmid DNA, diluted in sterile double distilled water to a final concentration of $0.2 \mathrm{mg} / \mathrm{ml}$, with $5 \mu \mathrm{l}$ of $2.5 \mathrm{M} \mathrm{CaCl}_{2}$ stock solution and $50 \mu \mathrm{l}$ of 2× BBS-buffered saline (50 mM BBS (N, N-bis[2-hydroxyethyl]-2-aminoethanesulfonic 
acid), $280 \mathrm{mM} \mathrm{NaCl}, 1.5 \mathrm{mM} \mathrm{Na}_{2} \mathrm{HPO}_{4}, \mathrm{pH}$ 7.05) kept at room temperature in darkness for 20 minutes. Mixture containing the precipitates was added drop-wise to $900 \mu \mathrm{l}$ NBA solution while gently vortexing. Neuronal cultures were taken out and moved to new wells, to each of which $500 \mu$ of the diluted calcium phosphate/DNA precipitate solution was added. After 20 -minute incubation at $37^{\circ} \mathrm{C}$ and $5 \% \mathrm{CO}_{2}$, a fine sandy precipitate covering the cells was observed. The cultures were then washed with prewarmed HBSS and finally replaced back to the original growth media. The efficiency was typically between 5 and $20 \%$, and there was no apparent toxicity to the cells. Imaging was performed at about $21 \mathrm{DIV}$.

Live Cell Immunostaining. In this study both cypHer5E-conjugated antibodies (mouse monoclonal $\alpha$ Syt1-cypHer 5E: $105311 \mathrm{cpH}$; rabbit polyclonal $\alpha$ VGAT-cypHer5E: $131103 \mathrm{cpH}$ ) were purchased from Synaptic Systems (Göttingen, Germany). Neurons were incubated in cell culture incubator for 3 hours with the staining buffer $(1: 200)$ containing $120 \mathrm{mM} \mathrm{NaCl}, 5 \mathrm{mM} \mathrm{KCl}, 2 \mathrm{mM} \mathrm{MgCl} 2,2 \mathrm{mM} \mathrm{CaCl}_{2}, 10 \mathrm{mM}$ glucose and 18 $\mathrm{mM} \mathrm{NaHCO}$. Before use, the stained neuronal culture was washed twice with antibodyfree medium. For $\mathrm{pH}$ titration measurements of cypHer, $\alpha$ Syt1-cypHer5E-stained neuronal culture was fixed with $4 \%$ paraformaldehyde (PFA) in phosphate buffered saline (PBS) at room temperature. $0.4 \%$ Saponin was used to permeabilize the cell membranes, making the internalized antibodies accessible to perfused buffers of different $\mathrm{pH}$. The selective staining of the surface-residual SV proteins was performed on the microscope stage by incubating the neurons with $\alpha$ Syt1-cypHer5E (1:50) in the presence of $1 \mu \mathrm{M}$ Tetrodotoxin (TTX) at room temperature for 5 minutes. The neurons within the region of interest were then constantly washed with Tyrode solution through perfusion capillary during the recording. 
Optical Measurement, Microscopy and Analysis. Coverslips were placed in a perfusion chamber containing a modified Tyrode solution (in mM: $120 \mathrm{NaCl}, 2.5 \mathrm{KCl}, 2 \mathrm{CaCl}_{2}, 2$ $\mathrm{MgCl}_{2}, 10$ Glucose, $10 \mathrm{HEPES}, \mathrm{pH}$ was adjusted to 7.4 by $\left.\mathrm{NaOH}\right)$. To stimulate neurons, an electric field stimulation were delivered via two platinum electrodes spaced at $10 \mathrm{~mm}$ with $1 \mathrm{~ms}$ pulses of $50 \mathrm{~mA}$ and alternating polarity applied by a constant current stimulus isolator (WPI A 385, World Precision Instruments); $10 \mu \mathrm{M}$ 6-cyano-7-nitroquinoxaline2,3-dione (CNQX) and $50 \mu \mathrm{M}$ D,1-2-amino-5-phospHonovaleric acid (AP5) were added to prevent recurrent activity. Fast solution exchanges were achieved through a two-barrel glass tubing perfusion system controlled by a piezo-controlled stepper device (SF778, Warner Instruments). Fluorephores were excited at $488 \mathrm{~nm}$ (for $\mathrm{spH}$ ) or $640 \mathrm{~nm}$ (for cypHer) with a computer-controlled monochromator (Polychrom IV, Till Photonics) and time lapse images were acquired using an electron-multiplying CCD camera (Andor iXon+ DU-897E-BV camera; Andor Technology) which was mounted on an inverted Nikon TE2000 microscope equipped with a $\times 60,1.2$ numerical aperture water-immersion objective and an FITC/Cy5 dual-band high performance filter set (AHF analysentechnik AG, Tübingen). Images were analyzed using self-written program in Matlab (MathWorks). Active boutons were localized using an automated routine in which a difference image is generated by subtracting an average of five frames before from after stimulation. Square regions of interest $\left(1.5 \times 1.5 \mu \mathrm{m}^{2}\right)$ were centered on these spots and average fluorescence profiles were then plotted. Fluorescence traces from individual synapses were normalized before calculating the average response.

Theoretical Basis for pH-based Optical Signals. By given $\mathrm{pH}$, indicators are in the equilibrium with free protons. Therefore instantaneous fluorescence intensity reflects the proportion of protonated molecules, which can be calculated by the following equation, 
where $\mathrm{pK}$ is the negative logarithm of the equilibrium constant for protonation and [Ind'] for the fraction of protonated molecules. During the exo-endocytic cycling, the change in the fraction of protonated molecules can be quantified by the following expression, due to the exposure of intra-vesicular molecules to extracellular environment or the reacidification of freshly retrieved vesicles.

$$
\Delta\left[\operatorname{Ind}^{-}\right]=\frac{1}{1+10^{7.4-p K}}-\frac{1}{1+10^{5.6-p K}}
$$

Photobleaching Correction for cypHer Signals. As cypHer molecule displays much less photo-stability compared to its prototype (Cyanine 5 dye), it is required to correct the intensity of time-lapse images properly for photo-bleaching. Considering that the cypHer molecules would be quenched and temporarily protected from photo-bleaching, while being exposed to extracellular medium, the kinetics of exo-endocytic cycling could be successfully isolated using a memorial deconvolution algorithm. It is assumed that the intensity decline due to photo-bleaching can be described as an exponential decay (see below), where $I(t)$ is the instantaneous observation of intensity. Practically, the decay constant $\lambda$ is able to be obtained from observations of intensity-time courses in blank experiments, using the same exposure time and acquisition frequency, which indeed showed monoexponential bleaching characteristics.

$$
\frac{d I(t)}{d t}=-\lambda I(t)
$$

Therefore, the instantaneous intensity change caused by exo-endocytosis can be determined iteratively by the following equation, where is the instantaneous intensity observed at interval $\mathrm{t}$ - 1. An example of the correction is shown in Figure 2.1. 


$$
I(t)=I(0)-\sum_{i=0}^{t-1} \lambda I(i)+\Delta I(t)
$$

Deconvolution Analysis. The normalized fluorescence decay of calibration response was used as the template for the deconvolution. To minimize the noise, the template was simplified to a single exponential decay function obtained from fitting. The deconvolution was then performed by dividing the template from the normalized fluorescence time course in the Fourier space using Matlab built-in function. 

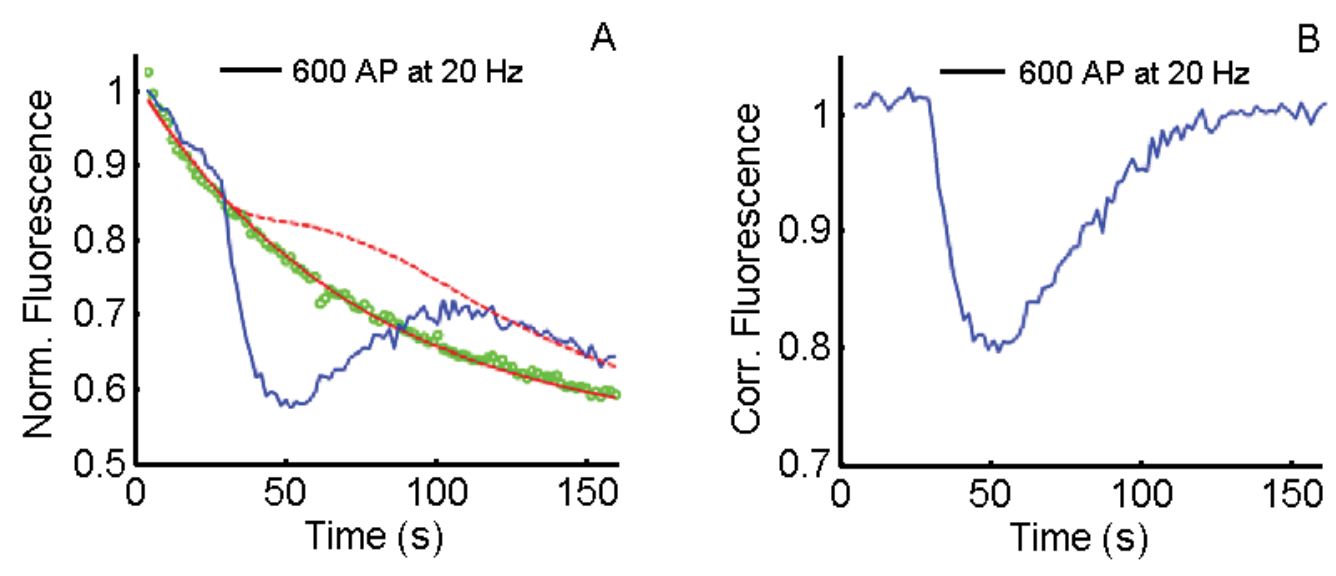

Figure 2.1 Photobleaching Correction for cypHer Signals

(A) During exo-endocytosis the fluorescence of cypHer is quenched and dequenched by $\mathrm{pH}$-change of the surrounding, which led to different photobleaching rates of different phases. An iterative correction is required to isolate the pure $\mathrm{pH}$-dependent fluorescence change. Average fluorescence time course of neurons stimulated by 600 APs was obtained by averaging the original data after normalization (blue). Standard bleaching curve (green circles) can be fit well to an exponential decay function (red line). The red dotted line indicates the working curve for the correction.

(B) Average cypHer fluorescence time course in (A) is plotted after correcting for photobleaching. Taken from (Hua et al., 2011).

Dual-color STED Microscopy. Dual-color super-resolution images were recorded with a custom-built confocal microscope using laser stimulated emission depletion (STED) technique. The setup combines two pairs of excitation and depleting laser beams all derived from a single supercontinuum laser source as described in (Liu et al., 2011). STED images were processed using a linear deconvolution function integrated into Imspector Software. The point spread function (PSF) for deconvolution was generated by using a 2D Lorentz function with its half-width and half-length fitted to the half-width and 
half-length of each individual image. Both Syt1 antibodies (mouse monoclonal, 604.2, Synaptic Systems, Göttingen, Germany) were directly labeled with either ATTO 647N or ATTO 590 and diluted (1:200) with Tyrode solution before use. For selective surface pool staining, a pre-incubation of neuron culture with $1 \mu \mathrm{M}$ TTX was required to effectively suppress spontaneous network activities. A detailed procedure of staining was described in the main text. After having been stained, sample was washed twice and fixed with $4 \%$ PFA at room temperature for 15 minutes and then mounted on a glass slice and transferred to the microscope stage for image acquisition. The colocalization of two Syt1 patches was quantitatively analyzed using Pearson correlation coefficients between signals from two channels after background subtraction. 


\section{Results}

\subsection{Surface Retrieval of SV Proteins}

To provide direct evidence for the stimulation-locked retrieval of surface-stranded vesicular proteins, a new assay was developed in our lab where the luminal domains of vesicular proteins are tagged with an exogenous $\mathrm{pH}$-indicator cypHer 5E (referred to here as cypHer) through antibodies (Adie et al., 2003; Martens et al., 2008). Protonation of this fluorophore can cause a five-fold increase in its quantum yield, internalization of labeled vesicular proteins will thereby lead to an increase in the fluorescence during reacidification inside the vesicle. To test this idea, antibodies against the luminal domain of synaptotagmin 1 (Syt1), and vesicular GABA transporter (VGAT) were labeled with cypHer and each separately applied to the neurons in culture. In both cases large accumulation of cypHer molecules in synaptic boutons were confirmed by a punctate staining due to dequenching of the fluorophores in the SV lumen (Figure 3.1).

Next, we attempted to monitor dynamics of surface residual vesicular proteins (This work was a collaboration with Raunak Sinha and has been published (Hua et al., 2011)). Brief incubation of the neuron culture in a saturating concentration of $\alpha$ Syt1-cypHer was conduct to selectively label the surface pool of vesicular proteins with limited dye internalization. $1 \mu \mathrm{M}$ tetrodotoxin (TTX) was added to the staining buffer to further reduce intermixing between the surface residual protein population and vesicular population through spontaneous activities by suppressing neural network activity (Groemer and Klingauf, 2007; Hua et al., 2010). When stimulated with 50 APs at $20 \mathrm{~Hz}$ after washout of TTX, the stained synaptic boutons responded with an increased fluorescence which gradually reached a plateau with a characteristic time constant, clearly 

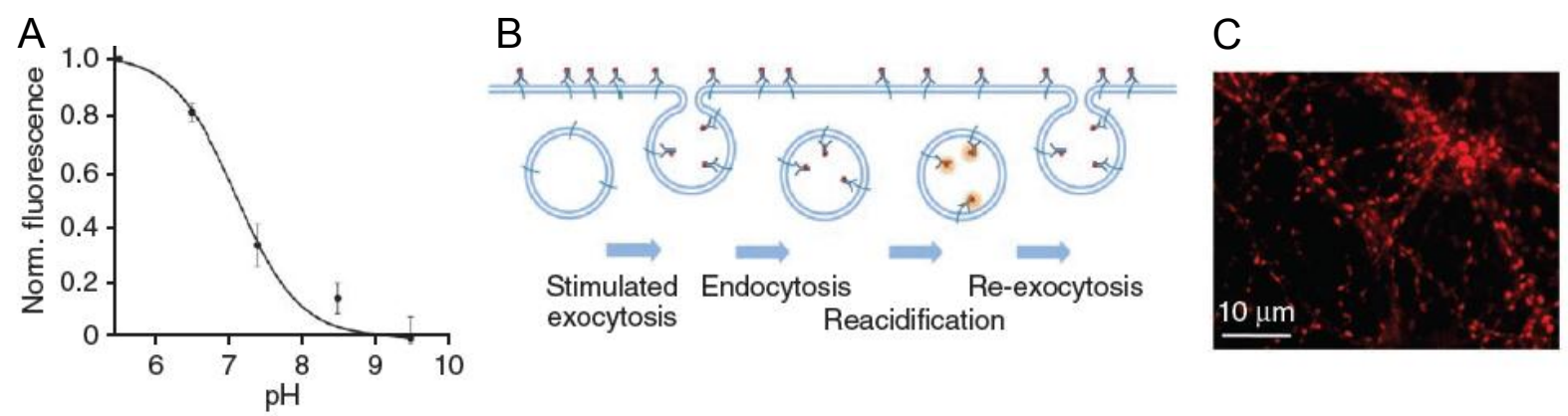

Figure 3.1 cypHer-coupled Antibodies Are a Reliable Tool to Measure Exoendocytic Cycling.

(A) Average normalized (norm.) fluorescence of anti-Syt1-cypHer-stained hippocampal boutons from multiple trials (each trials consisted of $>30$ boutons) as a function of $\mathrm{pH}$. The solid curve is a fit to a Henderson-Hasselbalch equation, yielding a pKa of 7.05. Error bars represent s.d.

(B) Schematic depicting fluorescence changes of cypHer during exo-endocytic cycling. The red dots show cypHer-coupled antibodies, which bind to the extracellular domain of SV proteins and remain quenched owing to the neutral extracellular $\mathrm{pH}$. During endocytosis and reacidification, however, the cypHer fluorescence is dequenched. Reexocytosis upon stimulations results in quenching of the cypHer fluorescence at the extracellular neutral $\mathrm{pH}$.

(C) Representative fluorescence image of hippocampal neurons labeled with anti-VGATcypHer. The label forms a punctate pattern with puncta typically representing individual boutons. Taken from (Hua et al., 2011)

indicating that stimulation triggers the retrieval of the RRetP to form fresh SVs (Figure 3.2 A). To further examine whether those SV recruited from the RRetP are functional and participate in vesicle cycling during repeated stimulation, a second round of stimulation was applied to surface Syt1-labeled neurons 60 seconds later. The fluorescence responses indeed now showed a fast drop in intensity due to partial exocytosis of previously recycled RRetP components again followed by an increase indicating endocytosis. The 
released fractions of SVs originating from the RRetP were $27.6 \pm 5.1 \%$ for 50 APs and $49.7 \pm 9.2 \%$ for 200 APs (Figure 3.2 B), which is in close agreement with similar experiments using FM styryl dyes as tracers (Vanden Berghe and Klingauf, 2006). However, unexpectedly, the fluorescence increase due to endocytosis after the second stimulus exceeded that of the exocytic quenching. This could be due to incomplete depletion of the RRetP during the first stimulus of 50 APs, but is more likely to be due to partial replenishment of the RRetP after the first stimulus by exocytosed vesicles that had taken up cypHer by spontaneous recycling during the initial labeling phase (Groemer and Klingauf, 2007; Hua et al., 2010).

A

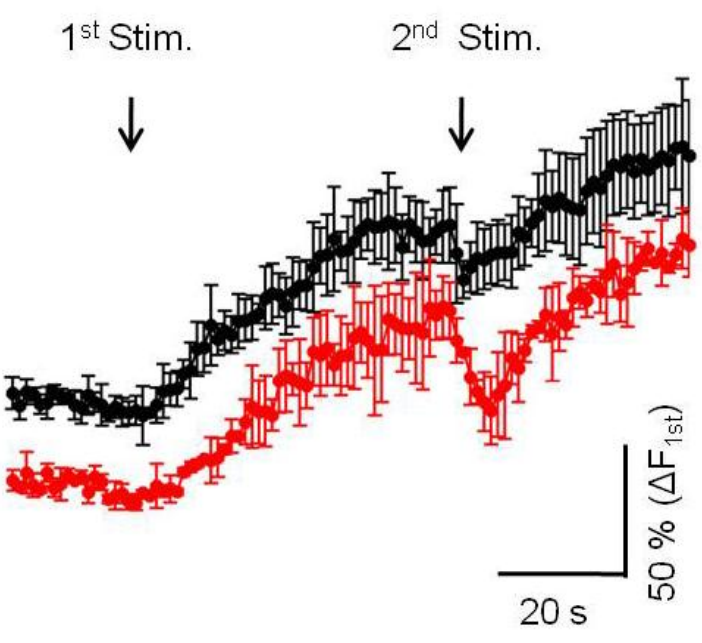

B

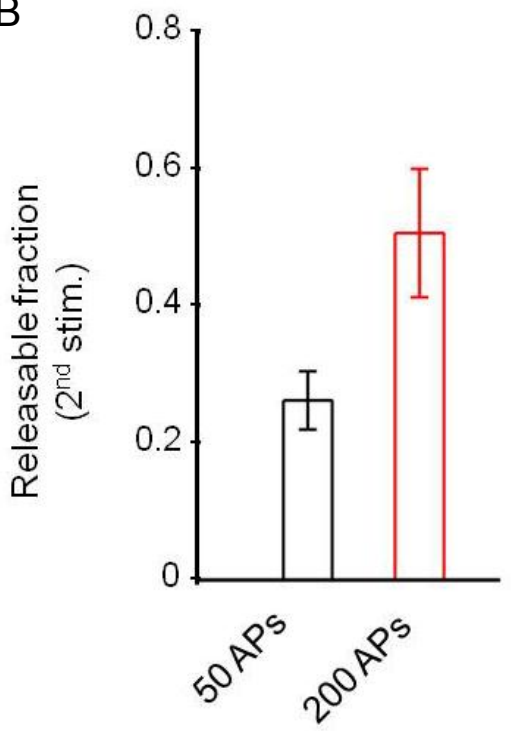

Figure 3.2 Readily Retrievable Surface Pool of SV Components.

(A) Average fluorescence profile of surface Syt1-labeled bouton ( $\mathrm{n}=3$ experiments with $>50$ boutons for each) stimulated first with 50 APs at $20 \mathrm{~Hz}$ followed by a second stimulation with either 50 (black) or 200 (red) APs at $20 \mathrm{~Hz}$. Error bar represent s.e.m.

(B) In response to the second stimulus, a marked fraction of SV recycled from the RRetP upon the first stimulation is released $(27.6 \pm 5.1 \%$ for 50 APs and $49.7 \pm 9.2 \%$ for 200 Aps). Taken from (Hua et al., 2011) 


\subsection{Exo-endocytosis Cycling of Endogenous SV Proteins}

Next, to clarify the question of whether fluorescence-labeled antibodies report same recycling pathway as $\mathrm{spH}$, neuronal cultures ware incubated with the cypHer-conjugated antibodies for three hours to label recycling vesicle during spontaneous neural activity so that recycling kinetics could be measured as fluorescence changes of cypHer upon electrical stimulation. For comparison, the staining was repeated in neurons transfected with $\mathrm{spH}$. The wide spectral separation enables dual-color imaging of $\mathrm{spH}$ and cypHer without bleed-through (Figure $3.3 \mathrm{~A}$ ). As the $\mathrm{spH}$ transfection efficiency is only $~ 10 \%$, not all $\alpha$ Syt1-cypHer-stained boutons express $\mathrm{spH}$. Therefore, automatic detection of active boutons was performed using spH responses and the created mask was then applied to cypHer channel. After having examined the fluorescence changes in response to 200 APs at $20 \mathrm{~Hz}$ in both channels, it was found that $\mathrm{spH}$ and cypHer responses of the same boutons showed mirror-image signals with near-identical endocytic time constants for monoexponential fits ( $\sim 23$ versus $\sim 22$ seconds). This indicates that overexpressed fusion proteins reliably report exo-endocytosis and do not artificially affect SV recycling.

In order to investigate the recycling kinetics of the inhibitory synapses, spH-transfected boutons were stained with $\alpha$ VGAT-cypHer (Figure $3.3 \mathrm{~B}$ ). A $~ 32 \%$ fraction of all spHexpressing boutons could be identified with $\alpha$ VGAT-cypHer, representing the fraction of inhibitory synapses in the cultures. Stimulation with 200 APs at $20 \mathrm{~Hz}$ induced similar fluorescence transients $(\sim 22$ seconds endocytic time constant) in both channels. Therefore, the kinetics of endocytosis and reacidification in inhibitory synapses are indistinguishable from the whole population response as probed with $\mathrm{spH}$ and cypHer antibodies. 


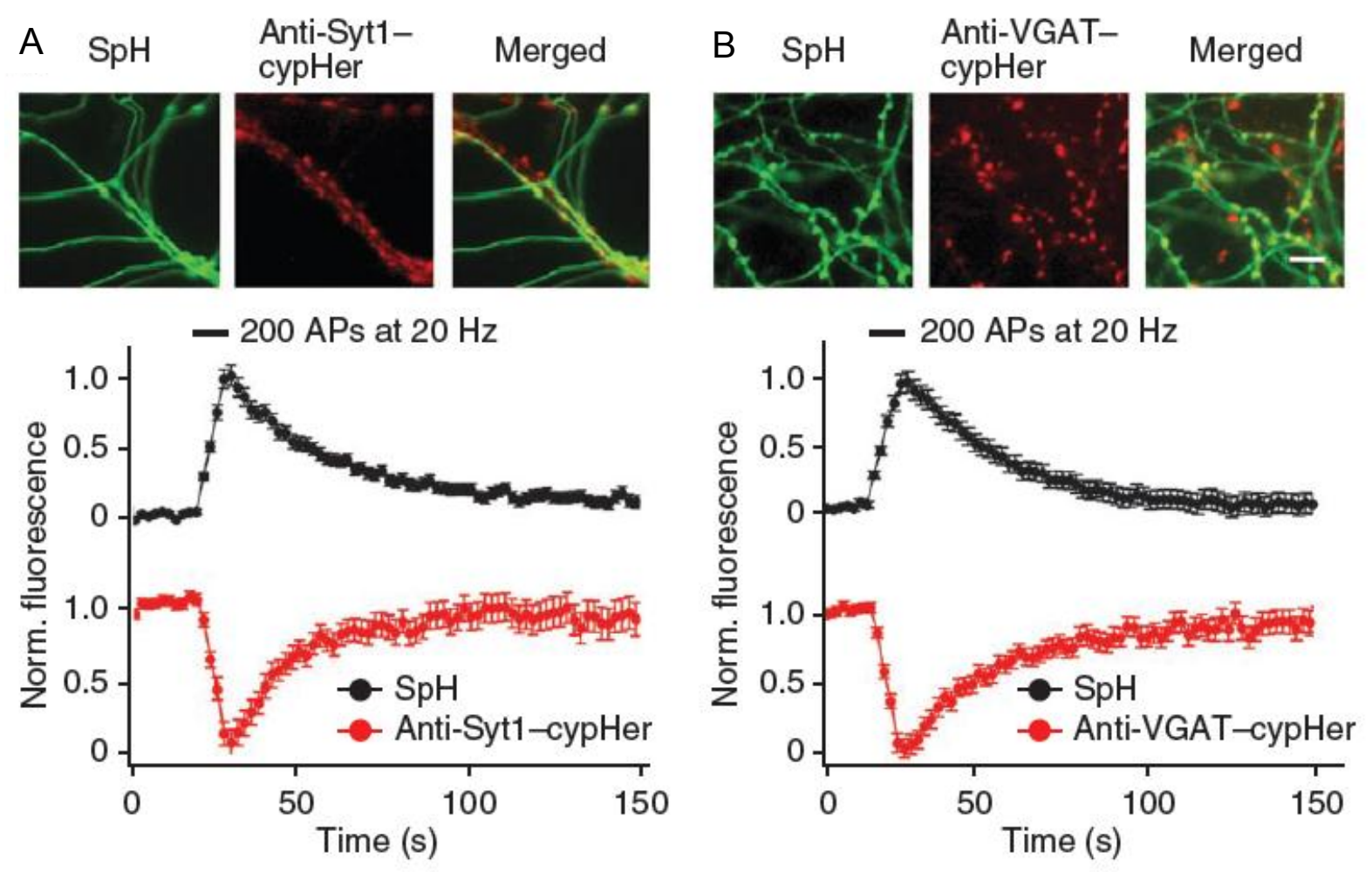

Figure 3.3 Comparison of Vesicle Recycling Kinetics Probed with spH and cypHercoupled Antibodies.

(A) Top: staining of spH-transfected hippocampal neurons with anti-Syt1-cypHer. Fluorescence images show colocalization of individual boutons expressing $\mathrm{spH}$ (green) with endogenous Syt1 (red) labeled with anti-Syt1-cypHer. Bottom: average fluorescence transients of double-labeled boutons in response to $200 \mathrm{APs}$ at $20 \mathrm{~Hz}$ ( $\mathrm{n}=$ 5 experiments with $>50$ boutons for each). Traces were corrected for photobleaching. Error bars represent s.e.m.

(B) Top: staining of spH-transfected hippocampal neurons with anti-VGAT-cypHer. Fluorescence images show colocalization of individual boutons expressing $\mathrm{spH}$ (green) with the inhibitory synapse marker VGAT (red). Bottom: average fluorescence transients of double-labeled boutons in response to 200 APs at $20 \mathrm{~Hz}(n=5$ experiments with $>50$ boutons for each). Traces were corrected for photobleaching. Error bars represent s.e.m. Scale bars represent $5 \mu \mathrm{m}$. Taken from (ua et al., 2011) 


\subsection{Spatial Organization of the Readily Retrievable Pool}

To address the question how is the functional surface pool spatially organized at the hippocampal synaptic bouton, we used the same antibody, $\alpha$ Syt1, to specifically label the surface pool, at $4{ }^{\circ} \mathrm{C}$ to minimize spontaneous activities (This work was a collaboration with Cora Thiel \& Roman Schmidt and has been published (Hua et al., 2011)). We fixed the cells immediately and identified synapses by colabeling with antibodies to bona fide cytoplasmic matrix of AZ (CAZ) or postsynaptic density (PSD) proteins. The spatial organization of the Syt1 surface pool was investigated using dual-color isoSTED nanoscopy (Schmidt et al., 2008). In the three-dimensional reconstruction of hippocampal en passant boutons from isoSTED image stacks (Figure 3.4), distinct Syt1 patches were found in the vicinity of the AZ.

From a large list of antibodies to CAZ elements, we obtained good signal-to-noise ratio only with antibodies for RIM1 and RIM2. The observed antibody fluorescence distribution was broad, extending up to $1 \mu \mathrm{m}$ along its long axis. AZ sizes of up to $700 \mathrm{~nm}$ have been documented in hippocampal synapses in electron microscopy of single sections (Schikorski and Stevens, 2001), as well as in recent full three-dimensional reconstructions (Staras et al., 2010). The larger patches measured in our experiment indicate that AZ sizes are indeed overestimated by labeling the large RIM proteins at the N-terminal zinc-finger domain.

To provide an additional fingerprint of synapses, we modified the staining by targeting the PSD opposing the AZ with antibodies to Homer1. This introduced a characteristic spatial separation between the two channels. We analyzed the distribution of the Syt1 patches with respect to Homer1 in each of the three bouton regions, as depicted in the histograms. The mean distance between Syt1 and Homer1 increased as the azimuth angle $\alpha$ between 
the estimated orientation of the axon and the Syt1-Homer1 connecting vectors approaches 0 or $\pm \pi$, owing to a higher probability of Syt 1 patches localized adjacent to the axolemma but not associated with Homer1. At other angles, the observed distances are smaller and reach minimum distances of about $100-300 \mathrm{~nm}$ for a bouton. This finding agrees well with a localization analysis of a large number of synapses in 4Pi microscopy (Huve et al., 2008; Kano et al., 2001). Considering this result together with our live cell imaging data, we therefore conclude for that the associated Syt1 patches constitute the preassembled RRetP. 


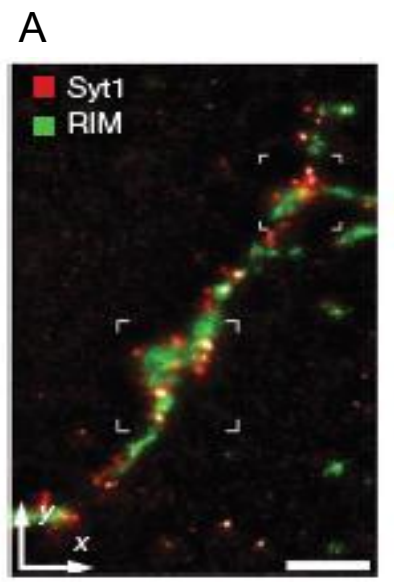

B

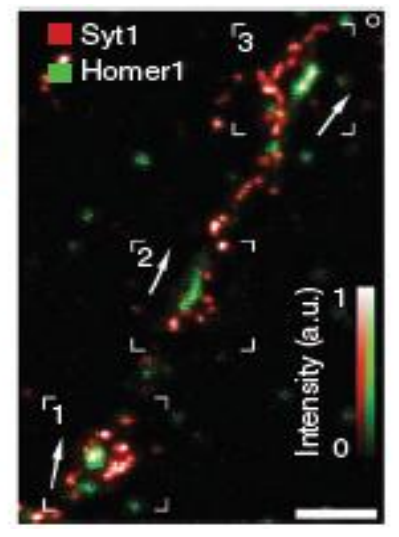

C

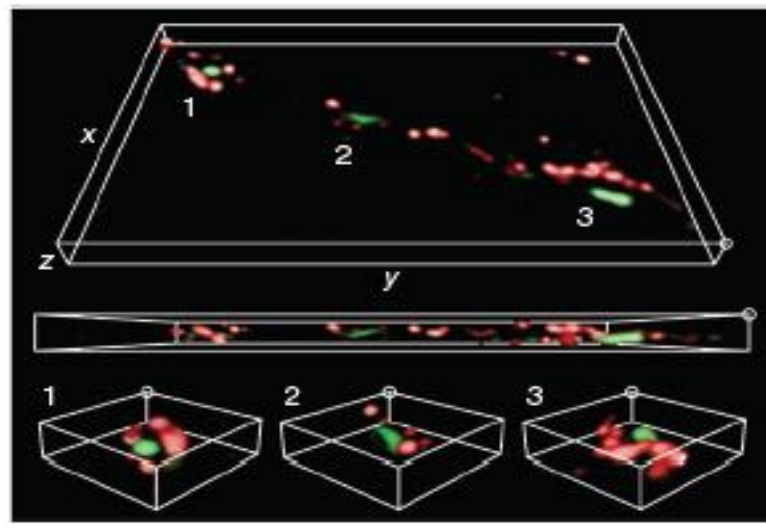

Region 3

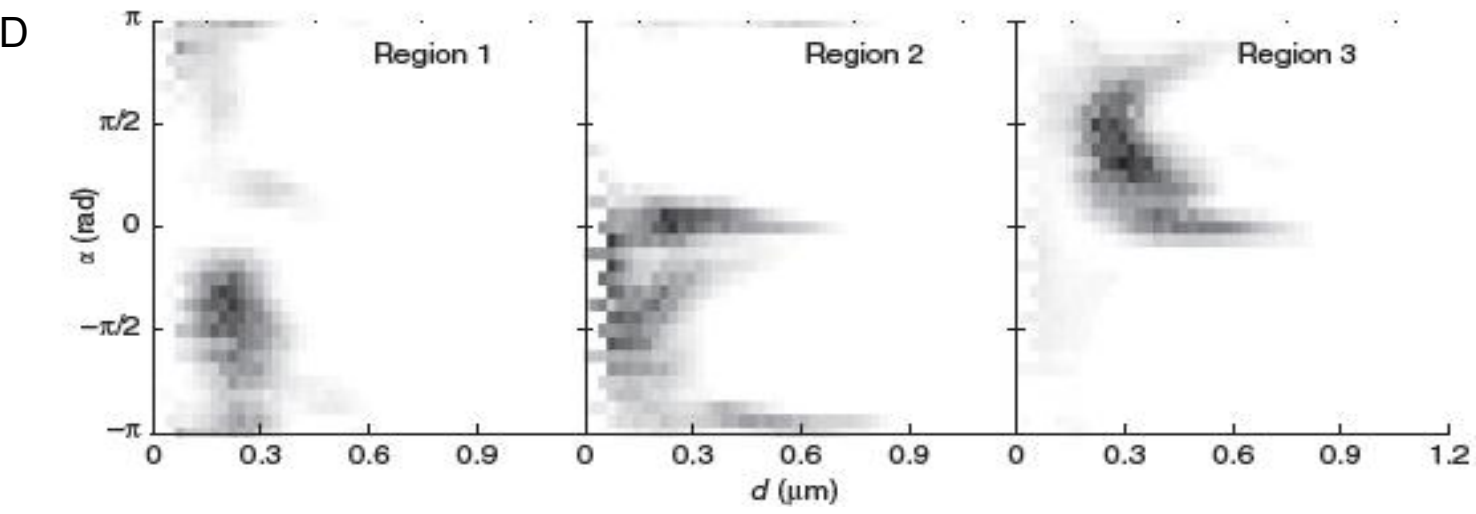

Figure 3.4 Spatial Organization of the RRetP

(A) Z-projection of a three-dimensional image stack of Syt1 at synapses labeled by the CAZ markers RIM1 and RIM2.

(B) Z-projection of Syt1 at synapses labeled by the PSD marker Homer1, with synapses at regions of interest numbered 1 and 3 . Arrows mark the local orientation of the axon.

(C) Perspective views of volume-rendered data. The Syt1 labeling reveals preassembled patches that are localized in the periphery of the AZ, typically within $500 \mathrm{~nm}$ distance from the postsynaptic marker.

(D) Spatial distribution of Syt1 with respect to Homer1 for each region of interest. Histograms show Syt1-Homer1 cross correlation, binned according to Syt1-Homer1 distance $d$ and relative azimuth angle $\alpha$, which was measured with respect to the orientation of the axon as marked by arrows in B. Distances between Syt1 patches and the postsynaptic marker accumulate at about 100-300 $\mathrm{nm}$ at angles where the probability of finding Syt1 patches that are not associated with Homer1 is reduced. Taken from (Hua et al., 2011) 


\subsection{Quantitative Measurement of Cumulative Release}

Spatial separation of the AZ and the endocytic site implies lateral diffusion of newly exocytosed patches of vesicular proteins from the release site towards the rim of the AZ where they are eventually retrieved. Therefore, sustained synaptic activities may cause an acute accumulation of vesicular proteins around the release site. This may eventually lead to a functional blockade of the previously used AZ slot, thereby causing STD as recently postulated (Neher, 2010). However, no study, to my knowledge, has been conduct to clarify whether unavailability of the release sites can become the rate limiting step during sustained synaptic activities.

To reliably measure the level of depression, attempts were made to quantify the amount of released vesicles upon different stimulation strengths using well established pHluorin assay. Under the same experimental conditions the absolute amplitude of fluorescence change is proportional to the amount of $\mathrm{spH}$ molecules in exocytosed vesicles and can differ from cell to cell due to inhomogeneous expression of the probe and variation in release probability of the synaptic bouton. Therefore, normalization of the recorded responses is required for quantitative measurements of release. However, in previous studies normalization of the fluorescence responses were performed either to the vesicle pool size or simply to the base-line fluorescence, neither of which correlates strongly to the factors discussed above (Burrone et al., 2006; Hua et al., 2010; Newton et al., 2006;

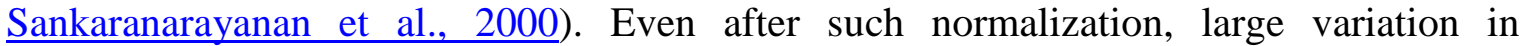
characteristic parameters of response remains, especially for small sample populations. To address this problem, an internal normalization reference was introduced by using a paired-stimulation protocol based on the fact that responses to brief stimuli remain stable over many trials when an interval of about 60 seconds is given for recovery after each stimulus (Burrone et al., 2006). In practice, a calibration stimulus which consisted of 50 
APs fired at $20 \mathrm{~Hz}$ was employed, since such a stimulus is believed to trigger the fusion of only docked vesicles without being affected by any downstream processes after exocytosis (Murthy and Stevens, 1999; Schikorski and Stevens, 1997). After a 60 second recovery interval, synapses were then stimulated with different test protocols. Each fluorescence transient from a given region of interest was normalized to the amplitude of the calibration stimulus response. Since the release probability and the average number of $\mathrm{spH}$ molecules per vesicle are conserved at the given synapse, their contribution to the variation between responses is negligible.

Using this method, I examined the effect of increasing stimulation frequencies on vesicle release and found that a higher level of normalized peak fluorescence could be reached for a given number of stimuli, if stimulation firing rate was increased (Figure 3.5). An obvious explanation for this is ongoing endocytosis during stimulation, which counteracts protein accumulation at the plasma membrane and therefore reduces the peak value of $\mathrm{spH}$ fluorescence. In order to compensate the effect of endocytosis and further characterize the role of stimulation frequency on release rate, attempts were made to quantify release rate and cumulative release from normalized fluorescence responses. First I extracted information about the kinetics of endocytosis and reacidification by fitting an exponential function to the decay phase of each calibration response. The vesicle retrieval time constant has been reported recently to be a cell-wide property and remains constant upon multiple rounds of trails for individual cells (Armbruster and Ryan, 2011). Therefore, assuming that the measured signal is a linear sum of elementary events, the relative release rate could be calculated by deconvolution of the decay from the normalized fluorescence transient. An increased endocytic time constant was constantly observed, whenever the cell was stimulated by 200 APs at more than $10 \mathrm{~Hz}$ (Figure 3.5), in line with previous work (Balaji et al., 2008). A potential explanation for such a progressive decline 
in endocytic rate is the exocytosis-driven protein accumulation on the surface that exceeds the buffering capacity of the RRetP. To test this idea, I explored the relationship between the stimulation strength versus the resulted endocytic time constant and found that at given stimulation frequency the endocytic rate remains constant until a given number of stimuli, beyond which the rate decreases rapidly (Figure 3.6). During low frequency stimulation, recovery of the RRetP may be fast enough to relieve the exocytic load and thereby ensure maximum speed of endocytosis for prolonged stimulation. This result confirms the existence of a limited surface capacity for fast membrane retrieval from the RRetP and suggests that the repopulation of this pool limits the rate of the endocytosis.
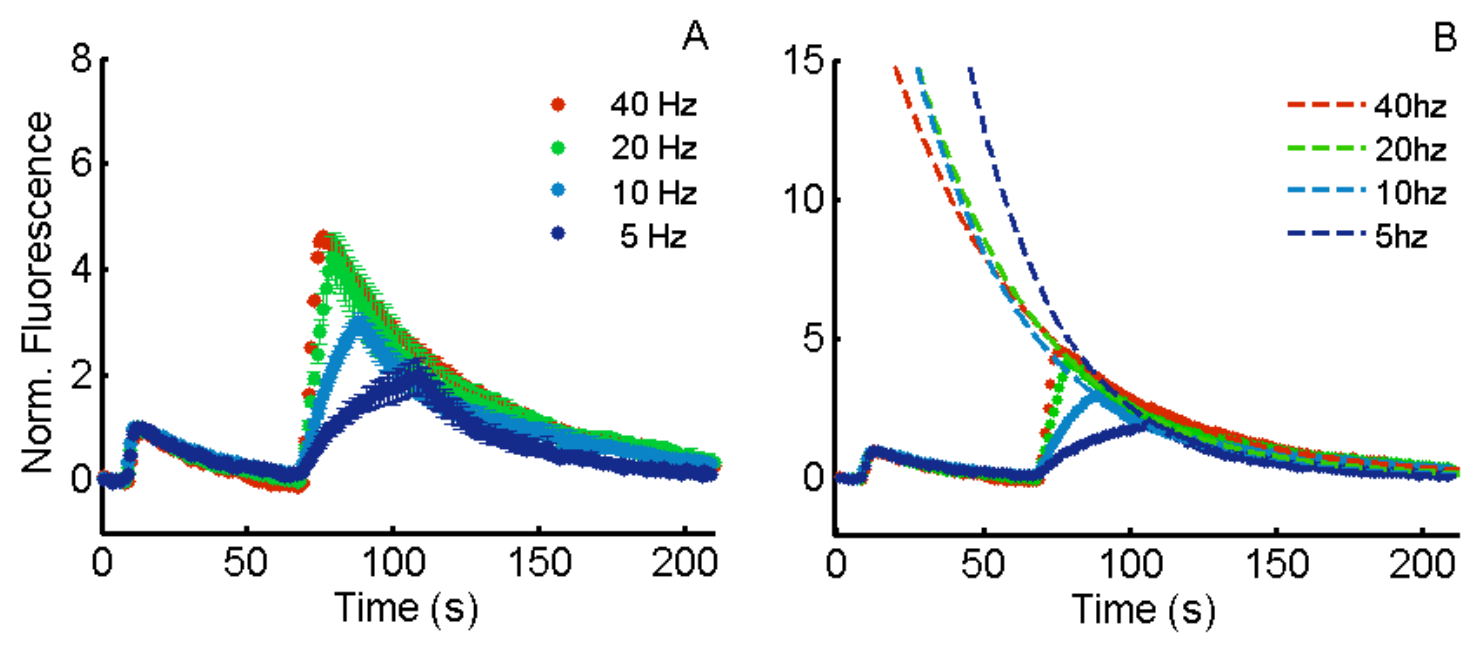

Figure 3.5 Normalization of spH Response Using a Paired-stimulation Protocol

(A) Average spH responses to $50 \mathrm{Aps}$ at $20 \mathrm{~Hz}$ followed by 200 Aps at 5 (blue), 10 (cyan), 20 (green) or $40 \mathrm{~Hz}$ (red) with $60 \mathrm{~s}$ of recovery in between ( $\mathrm{n}=5$ experiments with $>20$ boutons for each). Each curve was normalized to its peak value of the first response, decay of which was fit with an exponential function. The time constant was 16.1 s. Error bars represent s.e.m.

(B) The second responses of fluorescence transients from (A) were fit with a single exponential decay function, respectively. The estimated endocytic time constant increases with stimulation frequency: $16.6 \mathrm{~s}$ for $5 \mathrm{~Hz}, 18.3 \mathrm{~s}$ for $10 \mathrm{~Hz}, 20.5 \mathrm{~s}$ for $20 \mathrm{~Hz}$ and $23.1 \mathrm{~s}$ for $40 \mathrm{~Hz}$. 


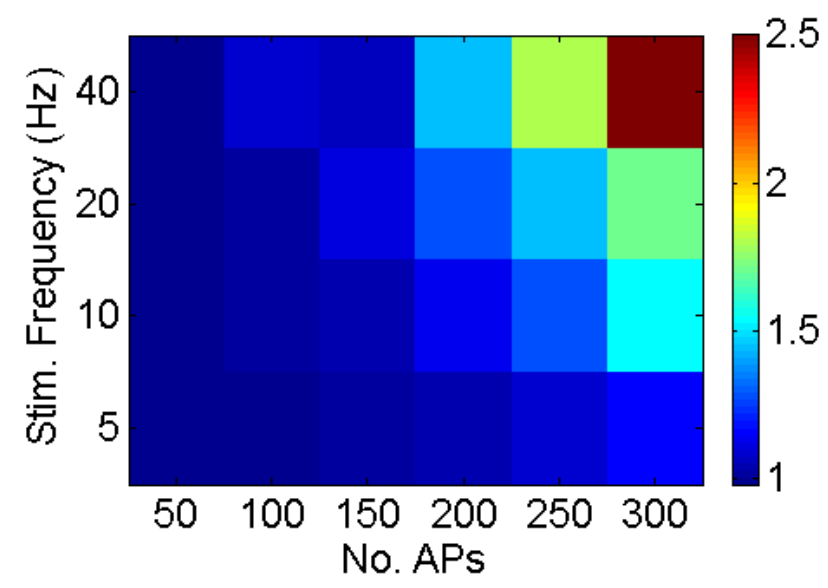

Figure 3.6 Color-coded Endocytic Time Constants of Synapses Challenged with Different Stimulation Protocols.

The fluorescence decay was fit by a single exponential function and obtained time constants were normalized to that of the calibration response evoked by 50 APs at 20 $\mathrm{Hz}$. High stimulation rate and prolonged stimulation lead to increased endocytic time constants.

When the endocytic load does not exceed the buffering capacity of the RRetP, the endocytic rate reaches its maximum and remains invariant, ensuring reliability of the outcome from the deconvolution method. This analysis revealed stepwise increases in release extending throughout the period of stimulation, indicating a linear release rate increase with mild stimulation up to $200 \mathrm{APs}$ at $5 \mathrm{~Hz}$, in agreement with several previous studies using alkaline trapping (Ariel and Ryan, 2010; Li et al., 2005). Integrating the release rate time course provided a good estimation of cumulative release for quantitative comparison (Figure 3.7). The resulting cumulative release was proportional to the number of APs up to 200, implying the absence of release depression during mild stimulation. 

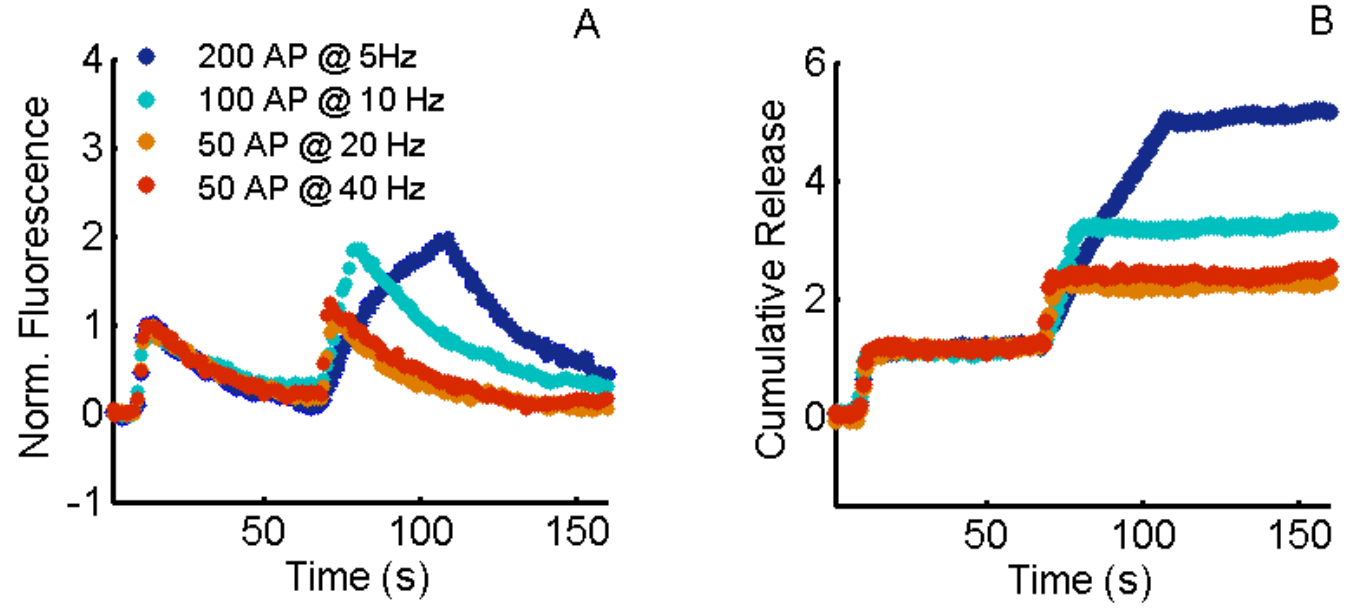

Figure 3.7 Quantitative Measurement of Cumulative Release

(A) Representative spH responses to 50 APs at $20 \mathrm{~Hz}$ followed by $200 \mathrm{APs}$ at 5 (blue), 100 at $10 \mathrm{~Hz}$ (cyan), 50 at $20 \mathrm{~Hz}$ (orange) or $50 \mathrm{~Hz}$ at $40 \mathrm{~Hz}$ (red) with $60 \mathrm{~s}$ of recovery in between. Each fluorescence transient from a given region of interest was normalized to the amplitude of the calibration stimulus response.

(B) Time courses of cumulative release calculated from (A). In normalized fluorescence transients of $(A)$, the decay phase of each calibration response was fitted with an exponential function. The relative release rate could be calculated by deconvolution of the decay from the fluorescence transient. Integrating the release rate time course provided a good estimation of cumulative release for quantitative comparison 


\section{5. $\quad$ Limited Vesicle Reuse during Mild Stimulation}

Despite its narrow range of applicability, the deconvolution method has an advantage over other methods that either block compensatory endocytosis or prevent vesicular reacidification, in fact it directly measures the rate of exocytosis without any perturbations. When applicable, it provides a better estimate for exocytosis, since it takes into account the contributions of fast vesicle reuse. In fact, comparing the results of deconvolution with that of using e.g. Folimycin (see below) should allow one to estimate the contribution of vesicle reuse.

I next compared the deconvolved-integrated signal with that from Folimycin (V-ATPase inhibitor) and Dynasore (dynamin GTPase inhibitor) experiments and found that during 200 AP stimulation firing at $5 \mathrm{~Hz}$ only the signal amplitude under Dynasore treatment was $\sim 9.2 \%$ smaller than that obtained from deconvolution (Figure 3.8). This difference suggests a minor contribution of fast reused vesicles to synaptic transmission or incomplete block by the agents. In agreement with several previous studies (Betz and Bewick, 1993; Li et al., 2005; $\underline{\mathrm{Wu} \text { and } \mathrm{Wu}, 2009)}$, this finding argues strongly against a major contribution of reused vesicles to synaptic transmission within a 40 second stimulation period. We cannot exclude the possibility of a kiss-and-run type of recycling pathway acting in addition, since fast opening and closing of fusion pores may only cause a relatively small $\mathrm{pH}$-fluctuation and therefore will not be fully reported by deconvolution (Zhang et al., 2009). 
A
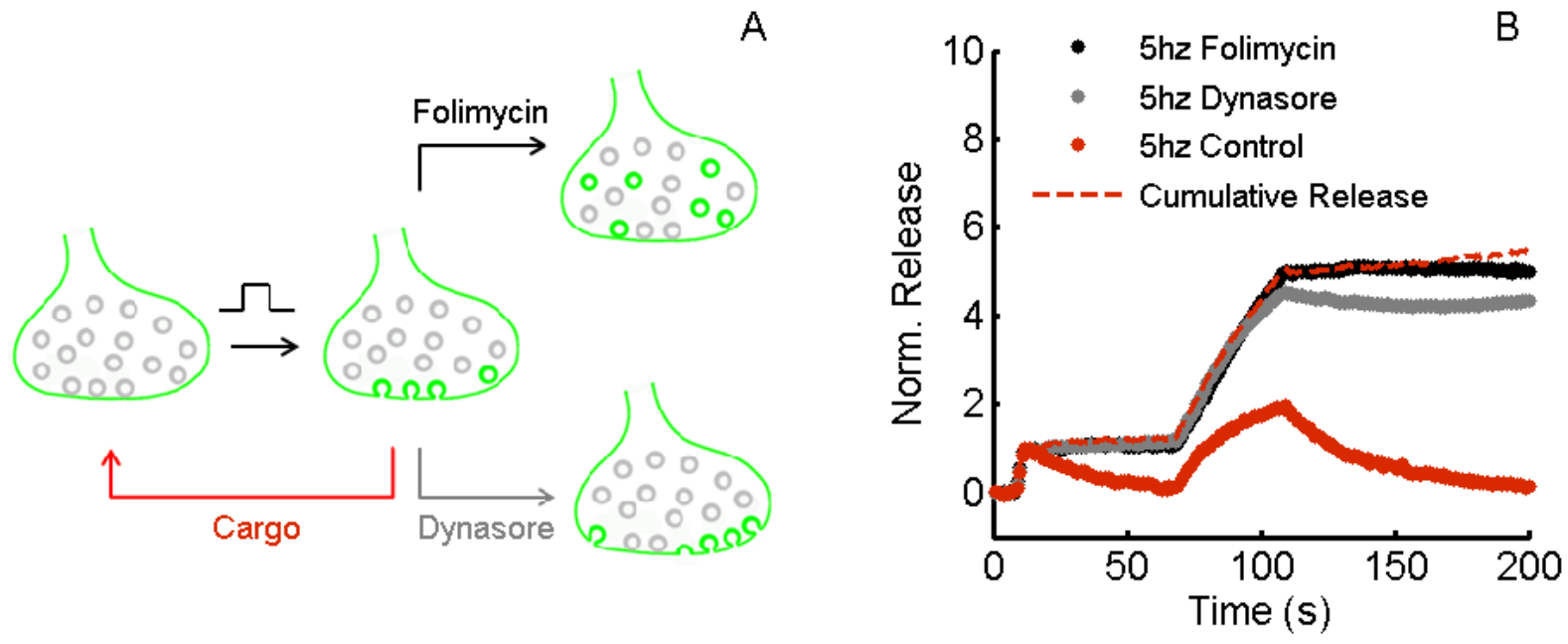

Figure 3.8 Same Amount of Vesicles Participating in Exocytosis during Mild Stimulation at $5 \mathrm{~Hz}$

(A) The basic experimental design is schematically illustrated.

(B) Comparison of the estimated cumulative release using three different methods. Same experiment like in Figure 3.7 was repeated in the presence of either Dynasore (gray) or Folimycin (black). Comparison of the deconvolved-integrated signal with that from Folimycin and Dynasore experiments revealed that during 200 APs firing at $5 \mathrm{~Hz}$ the signal amplitude from Dynasore-treatment was only $~ 9.2 \%$ smaller than that obtained from deconvolution. 


\subsection{Blocking Endocytosis Causes Rapid Release Depression}

As demonstrated above, synapses do not preferentially reuse freshly exocytosed vesicles during brief stimulation with low frequency (200 APs at $5 \mathrm{~Hz}$ ). When stimulating at frequencies of $10-100 \mathrm{~Hz}$, a reduction in synaptic response, known as STD, is observed in many types of glutamatergic synapses during electrophysiological recording. Upon sustained stimulation the rate of neurotransmitter release drops rapidly and reaches a steady state within 10-20 stimuli, reflecting a balance between vesicle usage and recruitment of new vesicles. To further examine the dynamics of vesicle cycling during stimulation with higher frequencies, I first repeated experiments using stimulation protocols similar to those of Figure $3.7 \mathrm{~B}$, but with increased frequency and in the presence of $65 \mathrm{nM}$ Folimycin (Fig $3.8 \mathrm{~A}$ ). Fluorescence amplitudes were found to be similar except for a slight drop for the highest stimulation frequency at $40 \mathrm{~Hz}$. The similarity of responses for 5, 10 and $20 \mathrm{~Hz}$ may suggest the absence of significant release depression and fast vesicle reuse. As sustained high release rate requires faster replenishment of vesicles in the RRP than that at rest, this result provides strong evidence for an activity-dependent replenishment mechanism to meet the needs of high release rate within a short period. This is not only in line with several previous studies (Dittman and

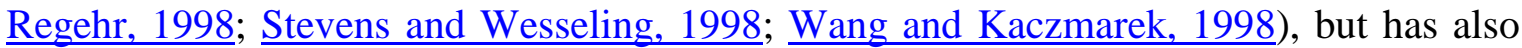
been further confirmed by the fact that a high-rate depleting pulse or increased extracellular $\mathrm{Ca}^{2+}$ concentration can drive more vesicles to participate in the exocytosis by enlarging the recycling pool size (Suppl. 1). 

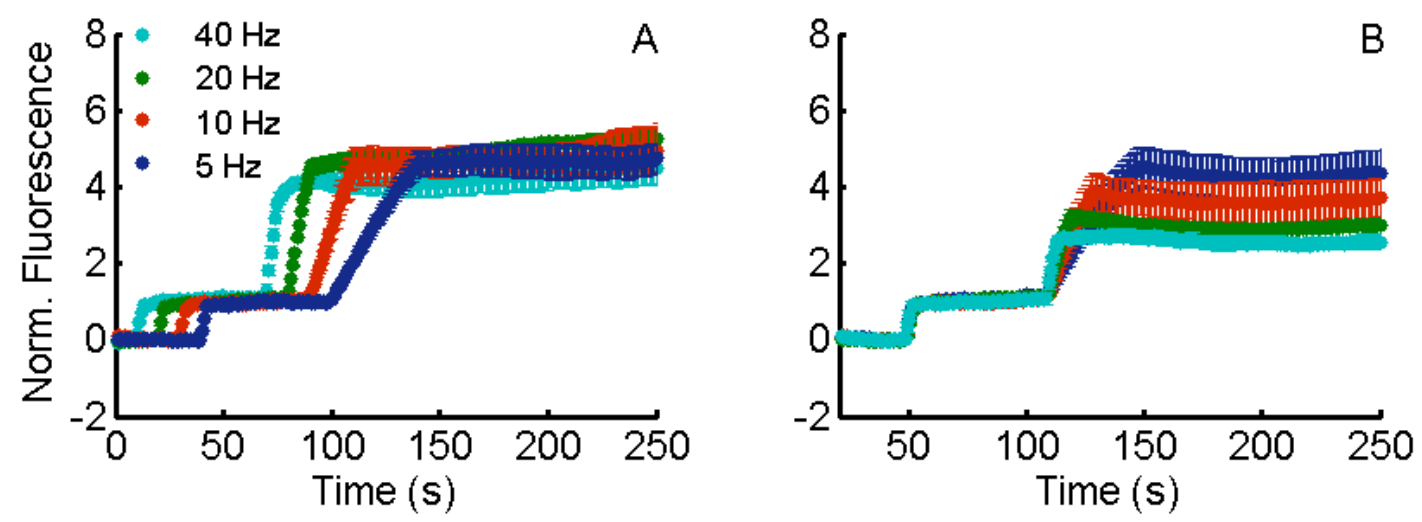

Figure 3.9 Frequency-dependent Release Depression in the Presence of Dynasore.

(A) Average $\mathrm{spH}$ responses to $50 \mathrm{APs}$ at $20 \mathrm{~Hz}$ followed by $200 \mathrm{APs}$ at 5 (blue), 10 (red), 20 (green) or $40 \mathrm{~Hz}$ (cyan) in the presence of Folimycin. Each fluorescence transient from a given region of interest was normalized to the amplitude of the calibration stimulus response. ( $n=6$ experiments with each $>20$ boutons for 5 and 40 $\mathrm{Hz} ; \mathrm{n}=6$ experiments for 10 and $20 \mathrm{~Hz}$ ). Fluorescence increases were insensitive to the stimulus frequency. Error bars represent s.e.m.

(B) Same experiments like in (A) were repeated in the presence of Dynasore ( $n=5$ experiments with $>20$ boutons for each). The amplitude of fluorescence responses dropped monotonically with increased AP-firing frequency. Error bars represent s.e.m.

Based on this finding I then tested whether STD is also enhanced after acute block of dynamin activity in primary neurons, as reported in the Calyx of Held (Hosoi et al., 2009). Indeed, when similar experiments were performed in the presence of $100 \mu \mathrm{M}$ Dynasore, the amplitude of fluorescence responses dropped monotonically with increased AP-firing frequency (Figure 3.9). This suggests that release depression during high frequency stimulation after block of dynamin activity is not a unique feature of large nerve terminal like Calyx of Held, but maybe a common property of excitatory synapses. The same experiment was repeated using cypHer as the pH-indicator (Hua et al., 2011) and similar results confirmed that the observed effect is not due to genetic perturbation by using spH (Suppl. 2). To further confirm that the reduction in fluorescence amplitude was Dynasore- 
specific, I examined spH response upon 200 APs at $20 \mathrm{~Hz}$ in the presence of both Dynasore and Folimycin (Figure 3.10) and found that addition of Folimycin could neither rescue nor intensify the release depression caused by Dynasore.

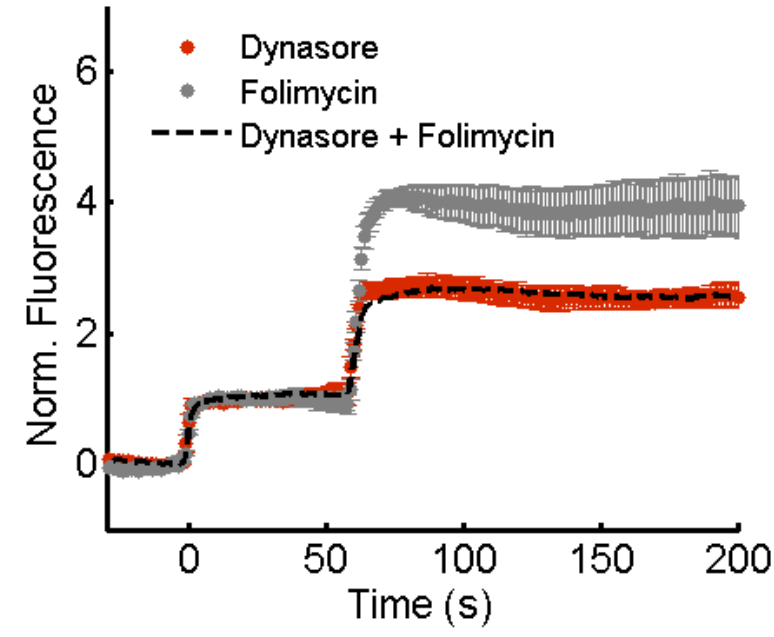

Figure 3.10. Reacidification has no Effect on Dynasore-enhanced Release Depression.

Average spH responses to 50 APs at $20 \mathrm{~Hz}$ followed by 200 APs at $40 \mathrm{~Hz}$ in the presence of Dynasore (red), Folimycin (gray) or both (black broken line). Between stimuli a $60 \mathrm{~s}$ interval was given for recovery. Each fluorescence transient was normalized to the amplitude of the calibration stimulus response. In the presence of both Folimycin and Dynasore resulted in same signal amplitude compared to that of only using Dynasore and about $38 \%$ smaller than that of using Folimycin. 


\subsection{Vesicle Recruitment during Multiple Spaced Stimuli}

When vesicles of the recycling pool are trapped in the alkaline state, mixing of these vesicles with the RRP will lead to a progressive reduction in the evoked fluorescence response, since fusion of alkaline-trapped vesicles does not contribute to the fluorescence increase. Such an effect was consistently observed during multiple rounds of short stimuli in the presence of a V-ATPase inhibitor ( $\mathrm{Li}$ et al., 2005). A similar phenomenon was also reported in hippocampal cultures treated with Dynasore (Newton et al., 2006), which was believed to be the consequence of vesicle pool depletion during sustained activity in the absence of dynamin-dependent endocytosis. To test and verify this idea, I analyzed responses of synapses expressing $\mathrm{spH}$ to multiple rounds of a brief stimulus (50 APs at 20 $\mathrm{Hz})$ at 60 second interval in the presence of either Dynasore, Folimycin or the delivery vehicle DMSO. Deconvolution was performed on control responses recorded with DMSO (Figure 3.11) and showed stepwise increases with identical amplitudes in cumulative release, ensuring the absence of major changes in synaptic function for at least 10 trials. When Folimycin was applied, fluorescence responses decreased gradually to $\sim 43 \%$ of the initial response, indicating an increased percentage of alkaline-trapped vesicles in the RRP. In the presence of Dynasore, however, there are no vesicles to be reused due to block of endocytosis and all release sites are occupied by fusion-competent vesicles recruited from a larger recycling pool. Similar response amplitudes were thereby expected at least during the initial few trials. Surprisingly, an accelerated reduction in responses was observed compared to that in the presence of Folimycin. This finding presented us with a puzzle about the mechanism behind the strong release depression caused by impaired dynamin activity even during the initial few trials. It is not expected that a small consumption of vesicles by exocytosis after few trials would affect the total number of recycling vesicles and cause strong release depression due to lack of release-ready 
vesicles. We therefore conclude that blocking of endocytosis by Dynasore must cause the enhanced STD by a mechanism other than insufficient vesicle supply.
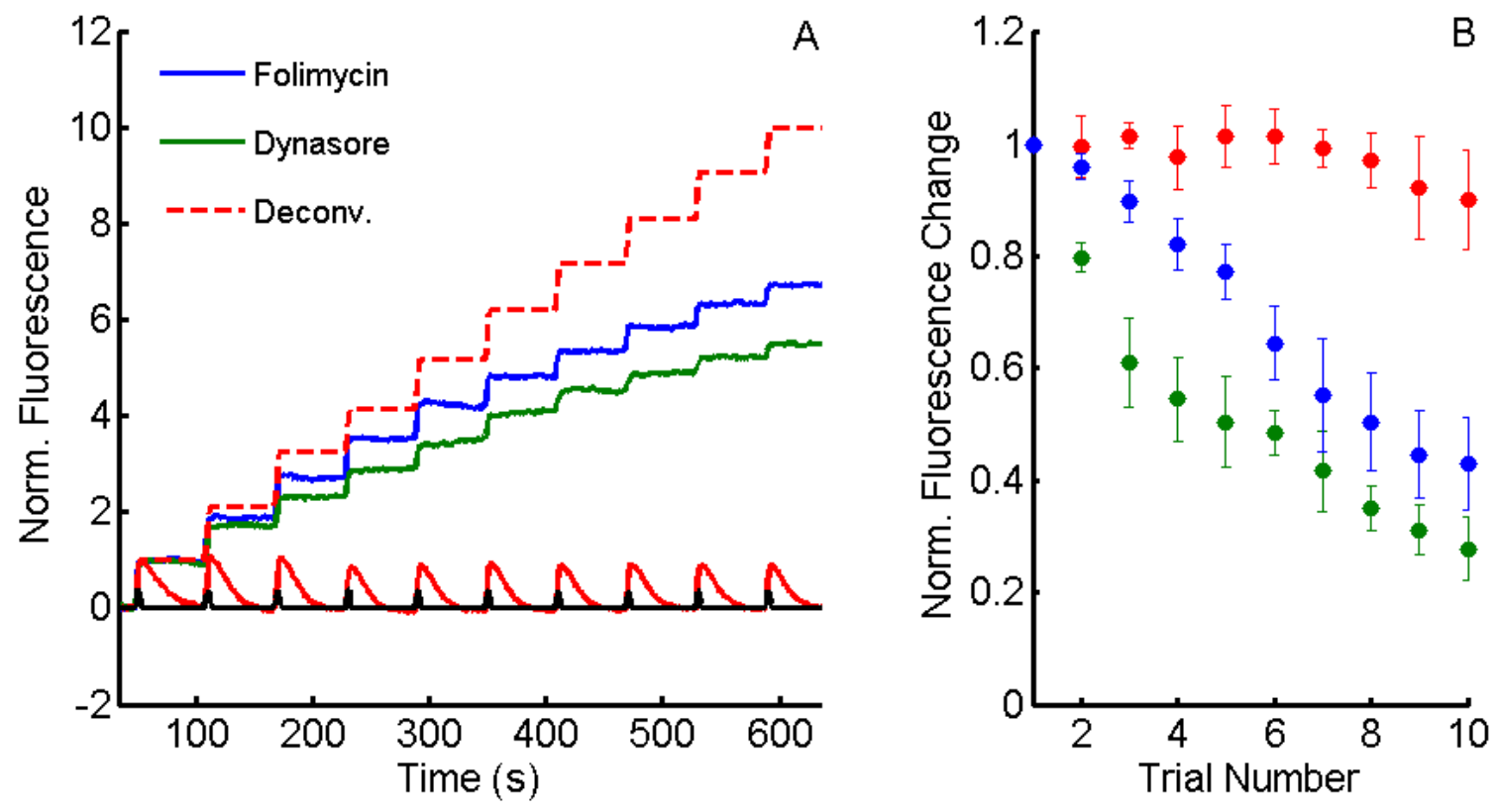

Figure 3.11 Recruitment of Reserve Vesicles during Multiple Spaced Stimuli

(A) Representative $\mathrm{spH}$ responses to consecutive runs of stimulation (50 APs at $20 \mathrm{~Hz}$ ) with 60 second interval for recovery (red solid line) plotted together with the calculated release rate (black) and cumulative release (read broken line). By comparison, same experiment was repeated in the presence of Folimycin (blue) or Dynasore (green). All fluorescence transients were normalized to the initial response, respectively.

(B) Plot of changes in fluorescence amplitudes upon each trial. In control experiment, evoked responses (red) remain almost constant over at least ten trials, while that of Dynasore (green) or Folimycin (blue) decreases with different rates ( $n=3$ experiments with $>20$ boutons for each). Error bars represent s.e.m. 


\subsection{Surface Accumulation of SV Components Caused by Dynasore}

The major phenotype of impaired dynamin activity has been shown to be a reduction in the vesicle pool size and the appearance of coated pits and invaginations at the stimulated synapses (Ferguson et al., 2007; Newton et al., 2006). An acute block of endocytosis or the loss of dynamin activity has been postulated to directly perturb the clearance of vesicle components from release sites, thereby interfering with release probability (Haucke et al., 2011; Kawasaki et al., 2000; Neher, 2010). Here I took the advantage of the newly developed super resolution STED microscopy technique to follow the fate of newly exocytosed vesicular proteins on the plasma membrane. Since Syt1, one of the vesicle associated proteins, is enriched at the periphery (potential endocytic site) of synapses at rest (see above), immunostaining of those surface residual proteins should indicate the location where exocytosed vesicle components will finally be retrieved (Hua et al., 2011). I first performed such surface staining using antibody coupled with red-colored fluorephore (ATTO $647 \mathrm{~N}$ ) on live hippocampal neuron culture, to which $1 \mu \mathrm{M}$ TTX was applied to suppress the network activities. After washing with normal saline, the culture was changed to another staining buffer containing the same Syt1 antibody coupled with green-shifted fluorephore (ATTO 590). The binding of ATTO 590 labeled antibody to the cells was completely blocked due to the absence of free epitopes on the surface, until the culture was stimulated with a train of $200 \mathrm{AP}$ at $20 \mathrm{~Hz}$ (Suppl. 3), which exposed unlabeled vesicular Syt1 to the surface. The detailed staining protocol is illustrated in Figure 3.12 A. Note that during the whole process Dynasore was present to block compensatory endocytosis, while control experiments were performed with DMSO. Two populations of Syt1 could thereby be well distinguished from each other by their colors using super resolution STED microscopy. Images revealed that Dynasore prevented newly exocytosed Syt1 patches from intermingling with the preexisting ones on the membrane 
(Fig 3.12 B). For a quantitative analysis the overlap of the two Syt1 populations was calculated using Pearson correlation coefficients between signals from two channels after background subtraction (Fig $3.12 \mathrm{C}$ ). Statistical significance in the difference between Dynasore and control groups argues strongly for a disruption in translocation of vesicular components towards endocytic sites. Together with reduced release probability observed under the same stimulation condition, this finding suggests a role of dynamin activity in the fast clearance of vesicular components from release sites, which is crucial for synapses to maintain high release rate during prolonged stimulation. 
A In the presence of $100 \mu \mathrm{M}$ Dynasore
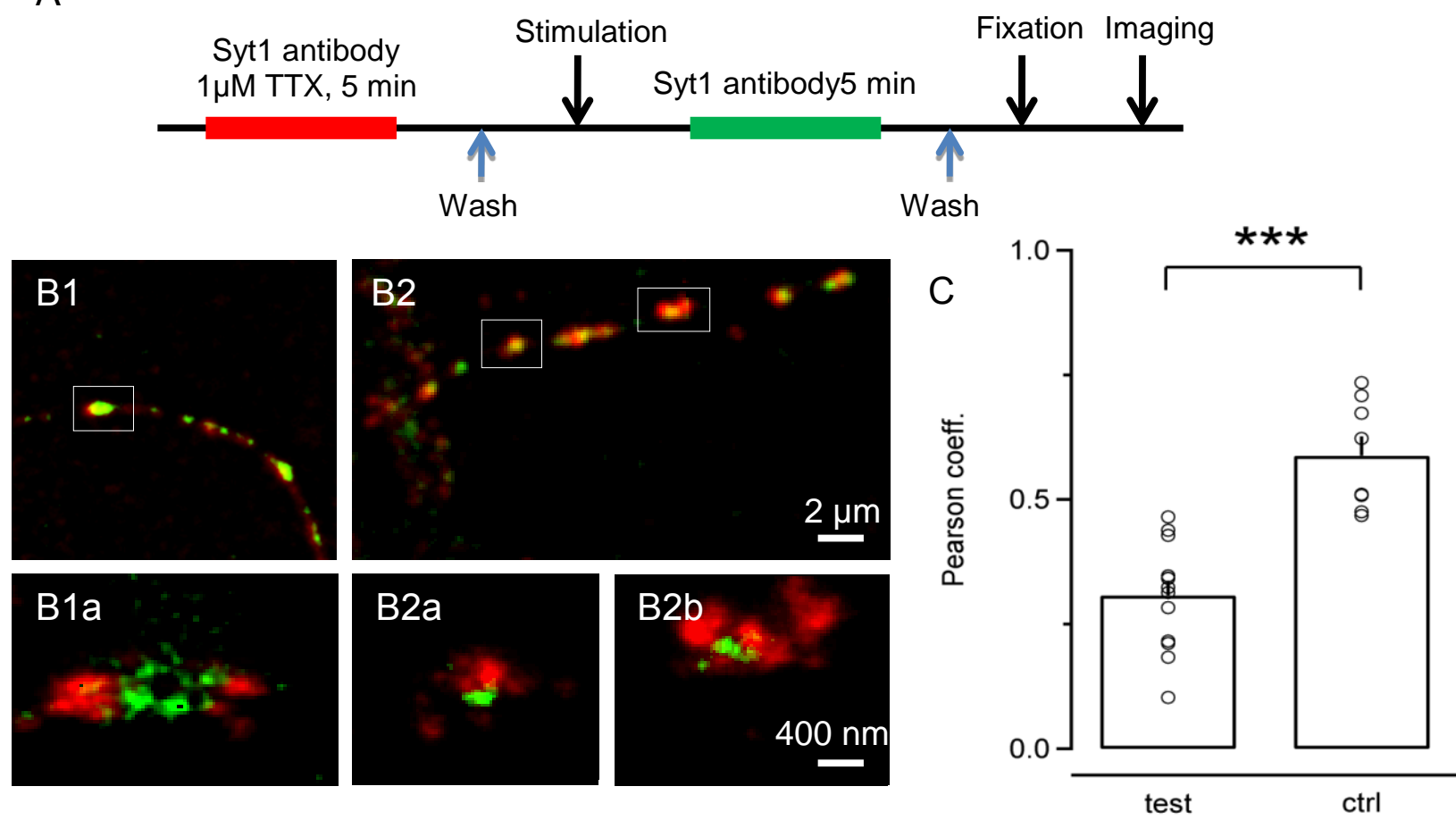

test

ctrl

Figure 3.12 Acuumulation of Vesicle Components in the Presence of Dynasore

(A) Experimental protocol for labeling surface-stranded and newly exocytosed Syt1 patches.

(B) Representive images of surface-stranded Syt1 (red) and newly exocytosed Syt1 (green). $100 \mu \mathrm{M}$ Dynasore was added to block compensatory endocyotsis. Overview was captured in the confocal mode (B1 and B2). Scale bar represents $2 \mu \mathrm{m}$. Images of $80 \mathrm{~nm}$ resolution were obtained by scanning synaptic boutons in the STED mode (B1a, B2a and B2b). Clear spatial separation between Syt1 patches was observed in STED images from experiments performed in the presence of Dynasore, indicating that Dynasore disrupts the translocation of newly exocytosed Syt1 patches towards RRetP. Scale bar represents $400 \mathrm{~nm}$.

(C) Statistic analysis of protein colocalization. Pearson coefficients were calculated for images from experiments performed either in the presence of Dynasore (test) or DMSO (control) after background subtraction. Significant reduction in Pearson coefficient was observed in Dynasore treated group compared to control group $(p<0.001, n=15$ experiments each). 


\section{Discussion}

During my $\mathrm{PhD}$, I have devised a new technique to measure synaptic activity in hippocampal synaptic boutons. Using cypHer-conjugated antibodies against luminal domains of Syt1 or VGAT, monitoring endogenous vesicle proteins during exo-endocytic cycling has achieved. Action-potential-driven fluorescence transients of $\alpha$ Syt1-cypHer and $\alpha$ VGAT-cypHer-stained synapses showed transients that were the mirror image of $\mathrm{spH}$ transients owing to their inverse $\mathrm{pH}$ dependence of cypHer fluorescence. Kinetics of endocytosis and reacidification assayed by both methods were nearly identical, showing that this cypHer-based approach reliably monitors recycling of endogenous synaptic vesicle proteins. The good spectral separation between cypHer and $\mathrm{spH}$ allowed us to use the two pH-dependent optical reporters in tandem, resulting in two independent readouts of presynaptic activity.

As previously shown, a significant percentage of SV proteins reside on the presynaptic membrane surface and SV interchange their membrane proteins with such surface reservoir during recycling (Fernandez-Alfonso et al., 2006). By bleaching or cleaving the pHluorin-tags of these surface SV proteins, it has been demonstrated that upon brief stimulation (40 APs) the vesicle proteins exo- and endocytosed lost their identity completely (Wienisch and Klingauf, 2006). To provide direct evidence for the active participation of RRetP-derived SVs in exo-endocytosis, I selectively labeled the RRetP with cypHer-conjugated antibodies and depleted the RRP by eliciting 50 Aps at $20 \mathrm{~Hz}$. The observed increase in cypHer fluorescence demonstrated preferential endocytosis of cypHer-stained Syt1 and VGAT residing on the presynaptic membrane rather than freshly exocytosed unlabeled ones. The existence of this functional pool of surface-stranded SV proteins is also corroborated by the nanoscopy of surface-stranded Syt 1 at the synaptic periphery, which is generally identified as the site of endocytosis. Images with a lateral 
resolution of $80 \mathrm{~nm}$ revealed several preassembled Syt1 patches, from which new synaptic vesicles could bud. Note that even for the low-copy-number protein VGAT, there was a functional surface pool that was preferentially internalized upon stimulated exoendocytosis, raising the question of whether this transporter might be active in the plasma membrane.

These findings demonstrate the existence of a functional RRetP of native vesicle constituents such as Syt1 and VGAT at the synaptic periphery. The preferential uptake of those vesicle constituents from the surface pool explains why an endocytosed vesicles is not composed of the most recently exocytosed vesicular proteins (Wienisch and Klingauf, 2006). Although overexpression of spH-tagged SV proteins may lead to a substantial increase of the total surface pool, the cypHer experiments clearly demonstrate the existence of an endogenous vesicular protein surface pool participating actively in SV cycling (Fernandez-Alfonso et al., 2006; Wienisch and Klingauf, 2006). Existence of such RRetP can explain the first wave of CME observed in the first reconstruction of the endocytic time course from electron micrographs of frog neuromuscular junctions (Miller and Heuser, 1984). Also, it provides an explanation for the reason why the initial endocytic rate remains constant irrespective of the stimulation strength, as suggested both in this and other studies (Balaji et al., 2008; Fernandez-Alfonso and Ryan, 2004; Sankaranarayanan and Ryan, 2000). However, after this RRetP is depleted, the rate of endocytosis drops because the endocytic machinery has to invest more energy and time to recapture and re-sort freshly exocytosed vesicle components to the sites of retrieval (Figure 3.7). Because endocytosis of the RRetP itself, however, takes several seconds, the main advantage of an RRetP might not be so much a gain in speed but rather a gain in precision of re-sorting and reclustering to ensure maximum fusion competence of newly endocytosed vesicles. The counterintuitive finding that knockdown or knockout of major 
sorting factors such as adaptor protein 1 (AP1) and AP2 subunits results in similar or even faster endocytic kinetics of a large fraction of retrieved SV proteins (Glyvuk et al., 2010; Kim and Ryan, 2009), can be brought in line with present investigations under the hypothesis that this fast endocytosis represents bulk endocytosis. The availability of endocytosed SVs is slowed and their fusion competence is diminished (Glyvuk et al., 2010) due to lack of a maturation step to an RRetP. In three recent studies, the importance of $\mathrm{Ca}^{2+}$ for coupling of exo- and endocytosis was emphasized (Hosoi et al., 2009; $\underline{\mathrm{Wu} \text { et }}$ al., 2009; Yao et al., 2009). Work in Drosophila suggested that there might be a vesicular $\mathrm{Ca}^{2+}$ channel that, once incorporated into the plasma membrane by fusion, would allow $\mathrm{Ca}^{2+}$ entry at the site of endocytosis and thus might trigger the retrieval from the RRetP (Yao et al., 2009). However, the exact molecular mechanism that couples exocytosis from the RRP with compensatory endocytosis from the RRetP and the regulatory factor of such pool still remains elusive.

So far this study demonstrated that exocytosed and endocytosed vesicles are driven in parallel from RRP and RRetP, respectively. In order to release neurotransmitter steadily at high rate, however, a mechanism is required to efficiently translocate newly exocytosed patches of vesicular proteins from the release site towards the rim of the $\mathrm{AZ}$ where the endocytic sites are located. Through examining the Dynsore-enhanced release depression using quantitative $\mathrm{spH}$ assay, I provide direct evidence for a novel mechanism that enhances STD, by functionally blocking the previously used AZ slot due to a release site clearance defect.

From technical point of view, I improved pHluorin-based assays for quantitative measurements of neurotransmitter release by using a novel normalization strategy of the $\mathrm{spH}$ response. The new protocol greatly reduces the variation in responses between synapses by applying a sequence of a standardized calibration stimulus followed by 
various test stimuli. With this method the inhomogeneity across synapses between responses to calibration and test stimulus is cancelled out. Furthermore, I combined this with a deconvolution method that provides an estimate of the cumulative release without any pharmacological perturbations. The deconvolution method distinguishes itself from alkaline-trapping by including the contribution of reused vesicles, which are not monitored by the latter method. Therefore, comparison between results from deconvolution with those from alkaline-trapping measurements serves as an excellent tool for quantifying vesicle reuse.

A surprising result in our data was the absence of preferential reuse of exocytosed vesicles under mild stimulation. I observed identical signal amplitudes for both deconvolution and alkaline-trapping in response to $200 \mathrm{APs}$ at $5 \mathrm{~Hz}$. Also, further experiments using Folimycin excluded the existence of additional vesicle reuse when firing frequency was increased up to $20 \mathrm{~Hz}$, while a small reduction in the recorded amplitudes during $40 \mathrm{~Hz}$ stimulation indicated some amounts of vesicle reuse, which may account for the reduction (Figure 3.9). Additionally the amount of release upon 200 APs appeared to be insensitive to stimulation frequency up to $40 \mathrm{~Hz}$, which may be a consequence of the activitydependent replenishment of RRP to meet the high demand of release-ready vesicles at AZ (Dittman and Regehr, 1998; Stevens and Wesseling, 1998; Wang and Kaczmarek, 1998). Moreover, I demonstrated that a high-frequency depleting pulse or elevated extracellular calcium concentration results in an increased recycling pool size (Suppl. 1). This suggests an accelerated replenishment that recruits vesicles from a release-reluctant pool rather than the slowly endocytosed vesicles. Taking this evidence together, I conclude that fast RRP replenishment alone can guarantee a sufficient vesicle supply during short period of physiological stimulation without additional contribution of rapid vesicle reuse. 
The measured cumulative release upon different stimulation conditions (Figure 3.10) provided quantitative information about how many vesicles can be eventually exocytosed in response to a seconds-long electrical stimulus. Based on that knowledge I was able to probe the release depression caused by the acute block of dynamin activity. Consistent with previous work performed at Calyx of Held using capacitance measurement ( al., 2009), I found that in cultured rat hippocampal neurons the perturbation of dynamin function leads to a significant reduction in the cumulative release during sustained high frequency $(40 \mathrm{~Hz})$ stimulation. When the same number of stimuli was applied at low rate $(5 \mathrm{~Hz})$, release depression was almost undetectable. It has been postulated that insufficient vesicle supply accounts for the release depression in Dynasore-treated neurons (Newton et al., 2006), since depletion of fusion competent vesicles is a direct consequence of impaired endocytosis under dynamin blockade. However, two aspects of our results challenge this view. First, stimulation at $5 \mathrm{~Hz}$ in the presence of Dynasore does mobilize more vesicles for fusion than at $40 \mathrm{~Hz}$, implying that the release depression upon high frequency stimulation occurs before vesicle pool depletion. Second, alkaline-trapping experiments show that even during high frequency stimulation for up to few seconds, vesicles are mainly recruited from the pre-existing vesicle pool rather than originating from recently endocytosed vesicles. Note that in these experiments the drug was applied to the cell culture only five minutes before recording to avoid any possible chronic changes like e.g. depletion of vesicles in the synaptic bouton, as reported in other studies where genetic knockout of the protein was employed (Lou et al., 2012).

So far, these results demonstrate that dynamin is essential for a process that occurs between exo- and endocytosis with a time constant shorter than that of vesicle recruitment. It manifests itself rapidly after dynamin activity is abolished. As proposed in recent works, translocation of synaptic components from sites of exocytosis to a 
"periactive zone" may be a good candidate for this step (Haucke et al., 2011; Neher, 2010). This is supported by findings that perturbations of dynamin and several interacting proteins enhance short-term synaptic depression upon high frequency stimulation (Hosoi et al., 2009; Marie et al., 2004). These studies include intersectin which interact with the actin regulatory proteins nwASP (neural wiskott-Aldrich syndrome protein) and CDC42 (cell division control protein 42) (Pechstein et al., 2010). Therefore, perturbed dynamin function may lead to the accumulation of vesicle components around release sites, as I observed under the STED microscope (Figure 3.13). This may prevent previous used release sites from participating in the recruitment and the release of additional vesicles. From the physiological point of view, release site clearance as a hypothetical mechanism, which nicely explains the deep depression during sustained high frequency stimulation after interference with dynamin function. Free diffusion of the exocytosed vesicular proteins on the plasma membrane may account for the remaining slow restoration of the release sites, while the effect of $\mathrm{CDC} 42$ block may suggest that the cytoskeleton is involved in rapid site clearance. Moreover, it has also been reported by Gaffield and colleagues that during high-frequency stimulation of mouse motor nerve terminal, the preferred sites of endocytosis were found much closer to the release sites as compared to mild stimulation (Gaffield et al., 2009). If this is also the case for cultured hippocampal neurons, deeper depression may be expected for high-frequency stimulation because of protein crowding around the release sites. This may provide a reason why a 60 second interval between multiple stimuli failed to rescue the depression in the presence of Dynaosre, if reduced distances between sites for exo- and endocytosis further restrict the diffusion of the exocytosed vesicle components. These results broaden the physiological importance of intact dynamin function by showing it is essential for the proper function of the synapse during sustained activity (Hosoi et al., 2009). 
In conclusion, our study demonstrates that impaired dynamin activity leads to a novel form of short-term depression, which is not related to insufficient vesicle supply at the synaptic bouton, but is the result of slow clearance of vesicular components from the release sites. This finding implies an important role of dynamin for sustained synaptic transmission at high rates beyond its well-known role in mediating a late step of endocytosis. 


\section{Summary}

In the central nervous system, communication between nerve cells mainly occurs at synapses via chemical neurotransmitters. Upon stimulation the transmitters are released by $\mathrm{Ca}^{2+}$-triggered exocytosis of $\mathrm{SV}$ s from presynaptic terminal to the synaptic cleft, where they activate postsynaptic receptors. In order to repopulate the pool of synaptic vesicle for further rounds of release, vesicle proteins need to be resorted and retrieved from the surface by compensatory endocytosis.

In this study, both exogenous cypHer-based and genetically encoded GFP-based ( $\mathrm{spH}$ ) $\mathrm{pH}$-switchable reporters are used to investigate the vesicle cycling in cultured rat hippocampal neurons. Using cypHer-conjugated antibodies against luminal domains of vesicle proteins (e.g. Syt1 and VGAT), visualizing cycling of endogenous vesicle proteins has been achieved. In addition, by monitoring surface-stranded vesicle proteins, constituting the so-called readily retrievable pool (RRetP), using cypHer-antibody, it was clearly demonstrated that upon stimulation stained Syt1 residing on the presynaptic membrane rather than freshly exocytosed unlabeled ones are preferentially retrieved to form fresh vesicles, indicating an active participation of the RRetP pool in the vesicle cycling. Furthermore, the existence of this RRetP is also corroborated by the observation of surface-stranded Syt1 at the synaptic periphery, which is generally identified as the site of endocytosis. These evidences indicate that exocytosis and subsequent compensatory endocytosis of vesicles are driven in parallel from the RRP and the RRetP, respectively. Since the surface pool is repopulated by newly exocytosed vesicle components, fast translocation of newly exocytosed vesicle proteins from AZ towards the synaptic periphery is expected. To explore potential roles of this translocation in synaptic transmission, dynamin-inhibitor Dynasore was applied to induce protein crowding by 
blocking endocytosis. Under influence of this drug a clear release depression was observed during high frequency stimulation, which was, however, absent under control conditions. Based on this observation, it is concluded that functional block of previously used release sites rather than insufficient vesicle supply accounts for the fast release depression after blocking endocytosis. This finding implies an important role of dynamin for sustained synaptic transmission at high rates beyond its well-known role in mediating fission, a late step of endocytosis. 


\section{Supplementary Figures}
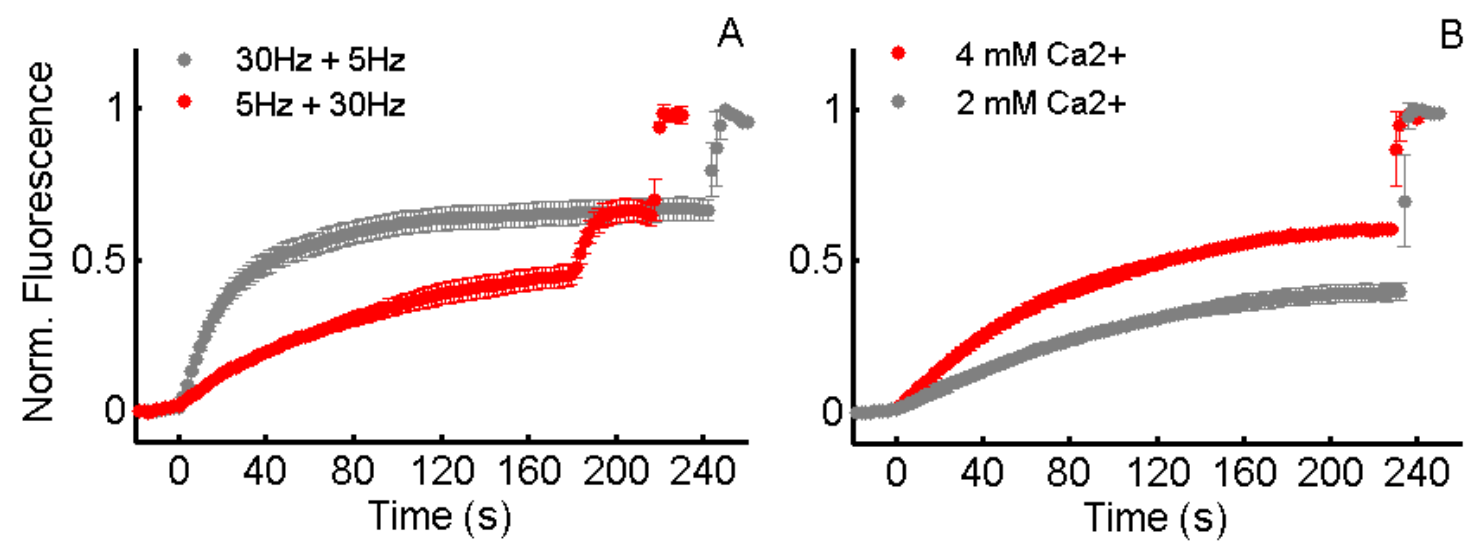

Suppl. 1 Calcium Level Regulates the Recycling Pool Size.

(A) Average spH response to two different depleting pulses in the presence of Folimycin. Vesicle pool was depleted either with a train of 1200 APs at $30 \mathrm{~Hz}$ followed by 1200 APs at $5 \mathrm{~Hz}$ (gray) or in an opposite order. A brief pulse of $\mathrm{NH}_{4} \mathrm{Cl}$ was applied at the end of the recording to determine the total vesicle population. Traces were normalized to the maximum dynamic range of $\mathrm{spH}$.

(B) Average spH response to $1200 \mathrm{APs}$ at $5 \mathrm{~Hz}$ in the presence of Folimycin. Experiments were conduct in a buffer containing either $2 \mathrm{mM}$ (gray) or $4 \mathrm{mM}$ calcium (red). A brief pulse of $\mathrm{NH}_{4} \mathrm{Cl}$ was applied at the end of the recording. Traces were normalized to the maximum dynamic range of $\mathrm{spH}$. 

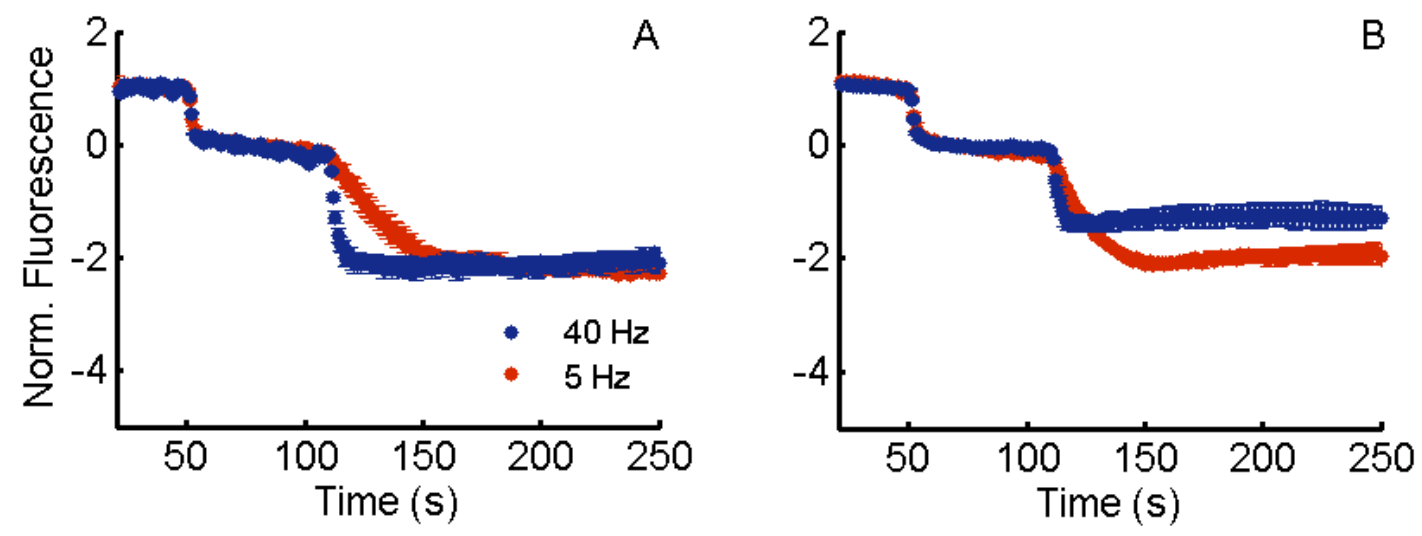

Suppl. 2 Dynasore-enhanced Release Depression Probed by cypHer

(A) Average cypHer responses to $50 \mathrm{APs}$ at $20 \mathrm{~Hz}$ followed by 200 APs stimulation at 5 (red) or $40 \mathrm{~Hz}$ (blue) in the presence of Folimycin. Each fluorescence transient from a given region of interest was normalized to the amplitude of the calibration stimulus response. ( $n=3$ experiments with $>20$ boutons for 5 and $40 \mathrm{~Hz}$ ). Error bars represent s.e.m.

(B) The same experiment as in (A), but was performed in the presence of Dynasore $(n=$ 3 experiments with $>20$ boutons for each). Each fluorescence transient from a given region of interest was normalized to the amplitude of the calibration stimulus response. Error bars represent s.e.m. 


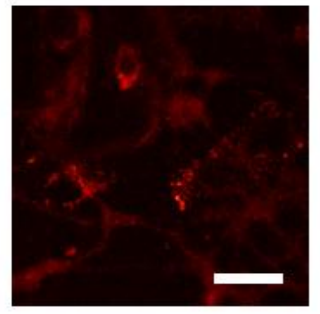

0 AP

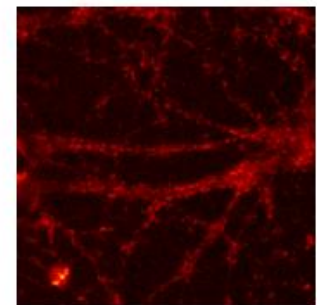

$50 \mathrm{AP}$

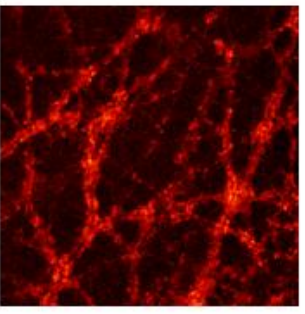

100 AP

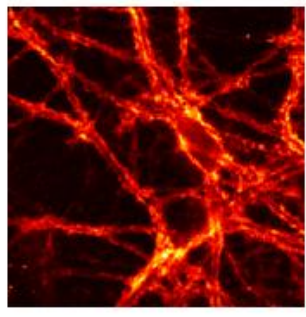

200 AP

\section{Suppl. 3 Live Staining of Newly Exocytosed SV Proteins}

(A) Representative images of neuron cultures stained with ATTO 647N labeled aSyt1antibodies. The cultures were preincubated with unlabeled antibodies to block surface residual epitopes and then challenged with stimulations of different APs at $20 \mathrm{~Hz}$ to evoke exocytosis. Dynasore was always present to block compensatory endocytosis. Without stimulation no signals of ATTO $647 \mathrm{~N}$ was observed (left), suggesting the absence of free epitopes on the surface, while fluorescence intensity increases with prolonged stimulation (middle, right), indicating that more epitopes become access to the antibodies due to exocytosis. Scale bar represents $10 \mu \mathrm{m}$. 


\section{Reference}

Adie, E.J., Francis, M.J., Davies, J., Smith, L., Marenghi, A., Hather, C., Hadingham, K., Michael, N.P., Milligan, G., and Game, S. (2003). CypHer 5: a generic approach for measuring the activation and trafficking of $G$ protein-coupled receptors in live cells. Assay and drug development technologies 1, 251-259.

Andreae, L.C., Fredj, N.B., and Burrone, J. (2012). Independent vesicle pools underlie different modes of release during neuronal development. J Neurosci 32, 1867-1874.

Aravanis, A.M., Pyle, J.L., and Tsien, R.W. (2003). Single synaptic vesicles fusing transiently and successively without loss of identity. Nature 423, 643-647.

Ariel, P., and Ryan, T.A. (2010). Optical mapping of release properties in synapses. Front Neural Circuits 4.

Armbruster, M., and Ryan, T.A. (2011). Synaptic vesicle retrieval time is a cell-wide rather than individual-synapse property. Nat Neurosci 14, 824-826.

Augustine, G.J., and Neher, E. (1992). Calcium requirements for secretion in bovine chromaffin cells. J Physiol 450, 247-271.

Balaji, J., Armbruster, M., and Ryan, T.A. (2008). Calcium control of endocytic capacity at a CNS synapse. J Neurosci 28, 6742-6749.

Balaji, J., and Ryan, T.A. (2007). Single-vesicle imaging reveals that synaptic vesicle exocytosis and endocytosis are coupled by a single stochastic mode. Proc Natl Acad Sci U S A 104, 2057620581.

Betz, W.J., and Bewick, G.S. (1993). Optical monitoring of transmitter release and synaptic vesicle recycling at the frog neuromuscular junction. J Physiol 460, 287-309.

Burrone, J., Li, Z., and Murthy, V.N. (2006). Studying vesicle cycling in presynaptic terminals using the genetically encoded probe synaptopHluorin. Nat Protoc 1, 2970-2978.

Dai, H., Shen, N., Arac, D., and Rizo, J. (2007). A quaternary SNARE-synaptotagmin-Ca2+phospholipid complex in neurotransmitter release. Journal of molecular biology 367, 848-863.

Daly, C., Sugimori, M., Moreira, J.E., Ziff, E.B., and Llinas, R. (2000). Synaptophysin regulates clathrin-independent endocytosis of synaptic vesicles. Proc Natl Acad Sci U S A 97, 6120-6125.

Deak, F., Schoch, S., Liu, X., Sudhof, T.C., and Kavalali, E.T. (2004). Synaptobrevin is essential for fast synaptic-vesicle endocytosis. Nat Cell Biol 6, 1102-1108.

Denker, A., Krohnert, K., Buckers, J., Neher, E., and Rizzoli, S.O. (2011). The reserve pool of synaptic vesicles acts as a buffer for proteins involved in synaptic vesicle recycling. Proc Natl Acad Sci U S A 108, 17183-17188.

Dittman, J., and Ryan, T.A. (2009). Molecular circuitry of endocytosis at nerve terminals. Annu Rev Cell Dev Biol 25, 133-160. 
Dittman, J.S., and Regehr, W.G. (1998). Calcium dependence and recovery kinetics of presynaptic depression at the climbing fiber to Purkinje cell synapse. J Neurosci 18, 6147-6162.

Doherty, G.J., and McMahon, H.T. (2009). Mechanisms of endocytosis. Annual review of biochemistry 78, 857-902.

Ferguson, S.M., Brasnjo, G., Hayashi, M., Wolfel, M., Collesi, C., Giovedi, S., Raimondi, A., Gong, L.W., Ariel, P., Paradise, S., et al. (2007). A selective activity-dependent requirement for dynamin 1 in synaptic vesicle endocytosis. Science 316, 570-574.

Fernandez-Alfonso, T., Kwan, R., and Ryan, T.A. (2006). Synaptic vesicles interchange their membrane proteins with a large surface reservoir during recycling. Neuron 51, 179-186.

Fernandez-Alfonso, T., and Ryan, T.A. (2004). The kinetics of synaptic vesicle pool depletion at CNS synaptic terminals. Neuron 41, 943-953.

Gaffield, M.A., Tabares, L., and Betz, W.J. (2009). Preferred sites of exocytosis and endocytosis colocalize during high- but not lower-frequency stimulation in mouse motor nerve terminals. $J$ Neurosci 29, 15308-15316.

Gandhi, S.P., and Stevens, C.F. (2003). Three modes of synaptic vesicular recycling revealed by single-vesicle imaging. Nature 423, 607-613.

Geppert, M., Goda, Y., Hammer, R.E., Li, C., Rosahl, T.W., Stevens, C.F., and Sudhof, T.C. (1994). Synaptotagmin I: a major Ca2+ sensor for transmitter release at a central synapse. Cell 79, 717727.

Glyvuk, N., Tsytsyura, Y., Geumann, C., D'Hooge, R., Huve, J., Kratzke, M., Baltes, J., Boening, D., Klingauf, J., and Schu, P. (2010). AP-1/sigma1B-adaptin mediates endosomal synaptic vesicle recycling, learning and memory. The EMBO journal 29, 1318-1330.

Granseth, B., Odermatt, B., Royle, S.J., and Lagnado, L. (2006). Clathrin-mediated endocytosis is the dominant mechanism of vesicle retrieval at hippocampal synapses. Neuron $51,773-786$.

Greengard, P., Valtorta, F., Czernik, A.J., and Benfenati, F. (1993). Synaptic vesicle phosphoproteins and regulation of synaptic function. Science $259,780-785$.

Groemer, T.W., and Klingauf, J. (2007). Synaptic vesicles recycling spontaneously and during activity belong to the same vesicle pool. Nat Neurosci 10, 145-147.

Harata, N., Pyle, J.L., Aravanis, A.M., Mozhayeva, M., Kavalali, E.T., and Tsien, R.W. (2001). Limited numbers of recycling vesicles in small CNS nerve terminals: implications for neural signaling and vesicular cycling. Trends Neurosci 24, 637-643.

Haucke, V., Neher, E., and Sigrist, S.J. (2011). Protein scaffolds in the coupling of synaptic exocytosis and endocytosis. Nat Rev Neurosci 12, 127-138.

Heuser, J.E., and Reese, T.S. (1973). Evidence for recycling of synaptic vesicle membrane during transmitter release at the frog neuromuscular junction. J Cell Biol 57, 315-344.

Hosoi, N., Holt, M., and Sakaba, T. (2009). Calcium dependence of exo- and endocytotic coupling at a glutamatergic synapse. Neuron 63, 216-229. 
Hua, Y., Sinha, R., Martineau, M., Kahms, M., and Klingauf, J. (2010). A common origin of synaptic vesicles undergoing evoked and spontaneous fusion. Nat Neurosci 13, 1451-1453.

Hua, Y., Sinha, R., Thiel, C.S., Schmidt, R., Huve, J., Martens, H., Hell, S.W., Egner, A., and Klingauf, J. (2011). A readily retrievable pool of synaptic vesicles. Nat Neurosci 14, 833-839.

Huve, J., Wesselmann, R., Kahms, M., and Peters, R. (2008). 4Pi microscopy of the nuclear pore complex. Biophys J 95, 877-885.

Jahn, R., and Scheller, R.H. (2006). SNAREs--engines for membrane fusion. Nat Rev Mol Cell Biol 7, 631-643.

Kano, H., Jakobs, S., Nagorni, M., and Hell, S.W. (2001). Dual-color 4Pi-confocal microscopy with 3D-resolution in the $100 \mathrm{~nm}$ range. Ultramicroscopy 90, 207-213.

Katz, B., and Miledi, R. (1965). Release of acetylcholine from a nerve terminal by electric pulses of variable strength and duration. Nature 207, 1097-1098.

Katz, B., and Miledi, R. (1967). Ionic requirements of synaptic transmitter release. Nature 215, 651.

Kavalali, E.T. (2007). Multiple vesicle recycling pathways in central synapses and their impact on neurotransmission. J Physiol 585, 669-679.

Kawasaki, F., Hazen, M., and Ordway, R.W. (2000). Fast synaptic fatigue in shibire mutants reveals a rapid requirement for dynamin in synaptic vesicle membrane trafficking. Nat Neurosci 3, 859-860.

Kim, S.H., and Ryan, T.A. (2009). Synaptic vesicle recycling at CNS snapses without AP-2. J Neurosci 29, 3865-3874.

Kim, S.H., and Ryan, T.A. (2010). CDK5 serves as a major control point in neurotransmitter release. Neuron 67, 797-809.

Klingauf, J., Kavalali, E.T., and Tsien, R.W. (1998). Kinetics and regulation of fast endocytosis at hippocampal synapses. Nature 394, 581-585.

Koenig, J.H., and Ikeda, K. (1996). Synaptic vesicles have two distinct recycling pathways. J Cell Biol 135, 797-808.

Kopp-Scheinpflug, C., Tolnai, S., Malmierca, M.S., and Rubsamen, R. (2008). The medial nucleus of the trapezoid body: comparative physiology. Neuroscience 154, 160-170.

Kwon, S.E., and Chapman, E.R. (2011). Synaptophysin regulates the kinetics of synaptic vesicle endocytosis in central neurons. Neuron 70, 847-854.

Lai, A.L., Huang, H., Herrick, D.Z., Epp, N., and Cafiso, D.S. (2011). Synaptotagmin 1 and SNAREs form a complex that is structurally heterogeneous. Journal of molecular biology 405, 696-706.

Li, H., Foss, S.M., Dobryy, Y.L., Park, C.K., Hires, S.A., Shaner, N.C., Tsien, R.Y., Osborne, L.C., and Voglmaier, S.M. (2011). Concurrent imaging of synaptic vesicle recycling and calcium dynamics. Frontiers in molecular neuroscience 4, 34. 
Li, Z., Burrone, J., Tyler, W.J., Hartman, K.N., Albeanu, D.F., and Murthy, V.N. (2005). Synaptic vesicle recycling studied in transgenic mice expressing synaptopHluorin. Proc Natl Acad Sci U S A 102, 6131-6136.

Liu, K.S., Siebert, M., Mertel, S., Knoche, E., Wegener, S., Wichmann, C., Matkovic, T., Muhammad, K., Depner, H., Mettke, C., et al. (2011). RIM-binding protein, a central part of the active zone, is essential for neurotransmitter release. Science 334, 1565-1569.

Lorteije, J.A., Rusu, S.I., Kushmerick, C., and Borst, J.G. (2009). Reliability and precision of the mouse calyx of Held synapse. J Neurosci 29, 13770-13784.

Lou, X., Fan, F., Messa, M., Raimondi, A., Wu, Y., Looger, L.L., Ferguson, S.M., and De Camilli, P. (2012). Reduced release probability prevents vesicle depletion and transmission failure at dynamin mutant synapses. Proc Natl Acad Sci U S A 109, E515-523.

Marie, B., Sweeney, S.T., Poskanzer, K.E., Roos, J., Kelly, R.B., and Davis, G.W. (2004). Dap160/intersectin scaffolds the periactive zone to achieve high-fidelity endocytosis and normal synaptic growth. Neuron 43, 207-219.

Martens, H., Weston, M.C., Boulland, J.L., Gronborg, M., Grosche, J., Kacza, J., Hoffmann, A., Matteoli, M., Takamori, S., Harkany, T., et al. (2008). Unique luminal localization of VGAT-C terminus allows for selective labeling of active cortical GABAergic synapses. J Neurosci 28, 13125-13131.

Martens, S., and McMahon, H.T. (2008). Mechanisms of membrane fusion: disparate players and common principles. Nat Rev Mol Cell Biol 9, 543-556.

Miesenbock, G., De Angelis, D.A., and Rothman, J.E. (1998). Visualizing secretion and synaptic transmission with pH-sensitive green fluorescent proteins. Nature 394, 192-195.

Miller, T.M., and Heuser, J.E. (1984). Endocytosis of synaptic vesicle membrane at the frog neuromuscular junction. J Cell Biol 98, 685-698.

Mohrmann, R., de Wit, H., Verhage, M., Neher, E., and Sorensen, J.B. (2010). Fast vesicle fusion in living cells requires at least three SNARE complexes. Science 330, 502-505.

Murthy, V.N., and De Camilli, P. (2003). Cell biology of the presynaptic terminal. Annu Rev Neurosci 26, 701-728.

Murthy, V.N., and Stevens, C.F. (1999). Reversal of synaptic vesicle docking at central synapses. Nat Neurosci 2, 503-507.

Neale, E.A., Bowers, L.M., Jia, M., Bateman, K.E., and Williamson, L.C. (1999). Botulinum neurotoxin A blocks synaptic vesicle exocytosis but not endocytosis at the nerve terminal. J Cell Biol 147, 1249-1260.

Neher, E. (2010). What is Rate-Limiting during Sustained Synaptic Activity: Vesicle Supply or the Availability of Release Sites. Front Synaptic Neurosci 2, 144.

Neher, E., and Sakaba, T. (2008). Multiple roles of calcium ions in the regulation of neurotransmitter release. Neuron 59, 861-872.

Newton, A.J., Kirchhausen, T., and Murthy, V.N. (2006). Inhibition of dynamin completely blocks compensatory synaptic vesicle endocytosis. Proc Natl Acad Sci U S A 103, 17955-17960. 
Nicholson-Tomishima, K., and Ryan, T.A. (2004). Kinetic efficiency of endocytosis at mammalian CNS synapses requires synaptotagmin I. Proc Natl Acad Sci U S A 101, 16648-16652.

Pechstein, A., Shupliakov, O., and Haucke, V. (2010). Intersectin 1: a versatile actor in the synaptic vesicle cycle. Biochem Soc Trans 38, 181-186.

Poskanzer, K.E., Marek, K.W., Sweeney, S.T., and Davis, G.W. (2003). Synaptotagmin I is necessary for compensatory synaptic vesicle endocytosis in vivo. Nature 426, 559-563.

Pyle, J.L., and Pelletier, M.S. (2003). Family and medical leave act: unresolved issues. New Solut $13,353-384$.

Rancz, E.A., Ishikawa, T., Duguid, I., Chadderton, P., Mahon, S., and Hausser, M. (2007). Highfidelity transmission of sensory information by single cerebellar mossy fibre boutons. Nature $450,1245-1248$.

Rizo, J., and Rosenmund, C. (2008). Synaptic vesicle fusion. Nat Struct Mol Biol 15, 665-674.

Rizzoli, S.O., and Betz, W.J. (2005). Synaptic vesicle pools. Nat Rev Neurosci 6, 57-69.

Rosenmund, C., and Stevens, C.F. (1996). Definition of the readily releasable pool of vesicles at hippocampal synapses. Neuron 16, 1197-1207.

Royle, S.J., Granseth, B., Odermatt, B., Derevier, A., and Lagnado, L. (2008). Imaging phluorinbased probes at hippocampal synapses. Methods Mol Biol 457, 293-303.

Ryan, T.A., Reuter, H., Wendland, B., Schweizer, F.E., Tsien, R.W., and Smith, S.J. (1993). The kinetics of synaptic vesicle recycling measured at single presynaptic boutons. Neuron 11, $713-$ 724.

Sakaba, T., and Neher, E. (2001). Calmodulin mediates rapid recruitment of fast-releasing synaptic vesicles at a calyx-type synapse. Neuron 32, 1119-1131.

Sankaranarayanan, S., De Angelis, D., Rothman, J.E., and Ryan, T.A. (2000). The use of pHluorins for optical measurements of presynaptic activity. Biophys J 79, 2199-2208.

Sankaranarayanan, S., and Ryan, T.A. (2000). Real-time measurements of vesicle-SNARE recycling in synapses of the central nervous system. Nat Cell Biol 2, 197-204.

Sankaranarayanan, S., and Ryan, T.A. (2001). Calcium accelerates endocytosis of vSNAREs at hippocampal synapses. Nat Neurosci 4, 129-136.

Schikorski, T., and Stevens, C.F. (1997). Quantitative ultrastructural analysis of hippocampal excitatory synapses. J Neurosci 17, 5858-5867.

Schikorski, T., and Stevens, C.F. (2001). Morphological correlates of functionally defined synaptic vesicle populations. Nat Neurosci 4, 391-395.

Schmidt, R., Wurm, C.A., Jakobs, S., Engelhardt, J., Egner, A., and Hell, S.W. (2008). Spherical nanosized focal spot unravels the interior of cells. Nature methods 5, 539-544.

Schweizer, F.E., and Ryan, T.A. (2006). The synaptic vesicle: cycle of exocytosis and endocytosis. Curr Opin Neurobiol 16, 298-304. 
Sinha, R., Ahmed, S., Jahn, R., and Klingauf, J. (2011). Two synaptobrevin molecules are sufficient for vesicle fusion in central nervous system synapses. Proc Natl Acad Sci U S A 108, 1431814323.

Smith, S.M., Renden, R., and von Gersdorff, H. (2008). Synaptic vesicle endocytosis: fast and slow modes of membrane retrieval. Trends Neurosci 31, 559-568.

Staras, K., Branco, T., Burden, J.J., Pozo, K., Darcy, K., Marra, V., Ratnayaka, A., and Goda, Y. (2010). A vesicle superpool spans multiple presynaptic terminals in hippocampal neurons. Neuron 66, 37-44.

Stevens, C.F., and Wesseling, J.F. (1998). Activity-dependent modulation of the rate at which synaptic vesicles become available to undergo exocytosis. Neuron 21, 415-424.

Sudhof, T.C. (2004). The synaptic vesicle cycle. Annu Rev Neurosci 27, 509-547.

Sudhof, T.C., and Rothman, J.E. (2009). Membrane fusion: grappling with SNARE and SM proteins. Science 323, 474-477.

Sun, J.Y., and Wu, L.G. (2001). Fast kinetics of exocytosis revealed by simultaneous measurements of presynaptic capacitance and postsynaptic currents at a central synapse. Neuron 30, 171-182.

Takamori, S., Holt, M., Stenius, K., Lemke, E.A., Gronborg, M., Riedel, D., Urlaub, H., Schenck, S., Brugger, B., Ringler, P., et al. (2006). Molecular anatomy of a trafficking organelle. Cell 127, 831846.

Takei, K., Mundigl, O., Daniell, L., and De Camilli, P. (1996). The synaptic vesicle cycle: a single vesicle budding step involving clathrin and dynamin. J Cell Biol 133, 1237-1250.

van den Bogaart, G., Holt, M.G., Bunt, G., Riedel, D., Wouters, F.S., and Jahn, R. (2010). One SNARE complex is sufficient for membrane fusion. Nat Struct Mol Biol 17, 358-364.

van den Bogaart, G., Thutupalli, S., Risselada, J.H., Meyenberg, K., Holt, M., Riedel, D., Diederichsen, U., Herminghaus, S., Grubmuller, H., and Jahn, R. (2011). Synaptotagmin-1 may be a distance regulator acting upstream of SNARE nucleation. Nat Struct Mol Biol 18, 805-812.

Vanden Berghe, P., and Klingauf, J. (2006). Synaptic vesicles in rat hippocampal boutons recycle to different pools in a use-dependent fashion. J Physiol 572, 707-720.

VogImaier, S.M., and Edwards, R.H. (2007). Do different endocytic pathways make different synaptic vesicles? Curr Opin Neurobiol 17, 374-380.

von Gersdorff, H., and Matthews, G. (1994). Inhibition of endocytosis by elevated internal calcium in a synaptic terminal. Nature $370,652-655$.

Wang, L.Y., and Kaczmarek, L.K. (1998). High-frequency firing helps replenish the readily releasable pool of synaptic vesicles. Nature 394, 384-388.

Wienisch, M., and Klingauf, J. (2006). Vesicular proteins exocytosed and subsequently retrieved by compensatory endocytosis are nonidentical. Nat Neurosci 9, 1019-1027.

Wu, W., Xu, J.H., Wu, X.S., and Wu, L.G. (2005). Activity-dependent acceleration of endocytosis at a central synapse. Journal of Neuroscience 25, 11676-11683. 
Wu, X.S., McNeil, B.D., Xu, J., Fan, J., Xue, L., Melicoff, E., Adachi, R., Bai, L., and Wu, L.G. (2009). $\mathrm{Ca}(2+)$ and calmodulin initiate all forms of endocytosis during depolarization at a nerve terminal. Nat Neurosci 12, 1003-1010.

$\mathrm{Wu}$, X.S., and Wu, L.G. (2009). Rapid endocytosis does not recycle vesicles within the readily releasable pool. J Neurosci 29, 11038-11042.

Yao, C.K., Lin, Y.Q., Ly, C.V., Ohyama, T., Haueter, C.M., Moiseenkova-Bell, V.Y., Wensel, T.G., and Bellen, H.J. (2009). A synaptic vesicle-associated Ca2+ channel promotes endocytosis and couples exocytosis to endocytosis. Cell 138, 947-960.

Young, S.M., Jr., and Neher, E. (2009). Synaptotagmin has an essential function in synaptic vesicle positioning for synchronous release in addition to its role as a calcium sensor. Neuron 63, 482-496.

Zhang, Q., Li, Y., and Tsien, R.W. (2009). The dynamic control of kiss-and-run and vesicular reuse probed with single nanoparticles. Science 323, 1448-1453. 


\section{Abbreviations}

\begin{tabular}{|c|c|}
\hline AP & Action Potential \\
\hline AP-1, AP-2 & Adaptor Protein-1,2 \\
\hline $\mathrm{AZ}$ & Active Zone \\
\hline CAZ & Cytoplasmic Matrix of Active Zone \\
\hline CME & Clathrin-mediated Endocytosis \\
\hline CNQX & 6-cyano-7-nitroquinoxaline-2,3-dione \\
\hline DIV & Day in Vitro \\
\hline DL-AP5 & D-amino-5-phosphonovaleric acid \\
\hline EPSC & Excitatory Postsynaptic Current \\
\hline HBSS & Hank's Balanced Salt Solution \\
\hline NBA & Neurobasal A Medium \\
\hline NSF & N-Ethylmaleimide-Sensitive Factor \\
\hline PFA & Paraformaldehyde \\
\hline PSD & Postsynaptic Density \\
\hline RIM 1/2 & Rab3-interacting Molecule 1/2 \\
\hline RRP & Readily Releasable Pool \\
\hline RRetP & Readily Retrieval Pool \\
\hline SNARE & Soluble NSF Attachment Protein Receptor \\
\hline $\mathrm{spH}$ & Synaptobrevin2-pHluorin \\
\hline STED & Stimulated Emission Depletion \\
\hline STD & Short-term Synaptic Depression \\
\hline STP & Short-term Synaptic Plasticity \\
\hline SV & Synaptic Vesicle \\
\hline TTX & Tetrodotoxin \\
\hline vGlut & Vesicular Glutamate Transporter \\
\hline VGAT & Vesicular GABA Transporter \\
\hline VGCC & Voltage-gated Calcium Channel \\
\hline
\end{tabular}




\section{Acknowledgement}

First of all I would like to thank my PhD supervisors Prof. Dr. Erwin Neher and Prof. Dr. Jürgen Klingauf for giving me the opportunity to work in their groups, for the motivation to undertake challenging projects and at the same time for being very patient mentors. Their openness to new ideas, the liberty to try novel experiments and the high level of enthusiasm about the result has made my $\mathrm{PhD}$ research a rich and a great learning experience.

I am grateful to the members of my thesis committee Dr. Alexander Egner for his valuable advice on my project and continuous support as an imaging expert.

I would like to acknowledge members of the Department of Nano-optics for a fruitful collaboration; Dr. Roman Schmid and Ulrike B for their help with isoSTED miscrospe. I would also like to thank Prof. Dr. Stefan Hell for his critical reading our manuscripts and sharing lab resource.

I would like to thank the past and present members of our department for creating a lively working environment. Thanks to Dr. Andrew Woehler for his help in imaging analysis and reading my thesis. I would like to express my gratitude to Prof. Dr. Takeshi Sakaba and Dr. Holger Taschenberger for regular discussions and advice on my project. Thanks to Dr. Cora Thiel for her help and advice in the initial phase of my PhD; Dr. Lijun Yao, Dr. Kun-Han Lin, Dr. Jin Bao, Dr. Chao-Hua Huang and Shu-Wen Chang for their help and support. I would like to specially thank my colleague Dr. Raunak Sinha for his great help with the cypHer projects. His outstanding experimental skills and solid knowledge in neuroscience steered two fruitful projects. 
Furthermore, I would like to acknowledge Michael Pilot, Ina Herfort for their expert technical assistance. I would also like to thank Frank Würriehausen for help with computers, Frank Köhne for help with electronics and Irmgard Barteczko for all the administrative support. 


\section{Curriculum Vitae}

\begin{tabular}{ll} 
Name: & \multicolumn{1}{l}{ Yunfeng Hua } \\
Nationality: & P.R. China \\
Date of birth: & 24 Jun. 1983 \\
Place of birth: & Shanghai, P.R. China \\
& \\
09.2001 - 06.2005: & B.Sc. in Chemistry \\
& Tongji-University, Shanghai, P.R. China \\
10.2005 - 03.2009: & M.Sc. in Chemistry \\
& Ludwig-Maximilians-Universität München, Germany \\
$03.2009-08.2012:$ & PhD student of Neurobiology \\
& Max Planck Institute for Biophysical Chemistry, Germany
\end{tabular} 


\section{Publication List}

Hua, Y.*, Sinha, R.*, Martineau, M., Kahms, M., and Klingauf, J. (2010). A common origin of synaptic vesicles undergoing evoked and spontaneous fusion. Nat Neurosci 13, 1451-1453. (* equal contribution)

Groemer, T.W., Thiel, C.S., Holt, M., Riedel, D., Hua, Y., Hüve, J., Wilhelm, B.G., Klingauf, J. (2011) Amyloid precursor protein is trafficked and secreted via synaptic vesicles PLoS One 6(4):e18754

Hua, Y.*, Sinha, R.*, Thiel, C.S.*, Schmidt, R., Huve, J., Martens, H., Hell, S.W., Egner, A., and Klingauf, J. (2011). A readily retrievable pool of synaptic vesicles. Nat Neurosci 14, 833-839. (* equal contribution)

Hua, Y., Woehler, A., Kahms, M., Klingauf, J., Neher, E. Blocking endocytosis enhances short-term synaptic depression under conditions of normal availability of vesicles. (Manuscript in preparation) 